\title{
Reactive microglia and IL1//LL-1R1- signaling mediate neuroprotection in excitotoxin-damaged mouse retina
}

\author{
Levi Todd ${ }^{6 \dagger}$, Isabella Palazzo ${ }^{1 \dagger}$, Lilianna Suarez ${ }^{1}$, Xiaoyu Liu, Leo Volkov², Thanh V. Hoang ${ }^{3}$, Warren A. Campbell ${ }^{1}$,
} Seth Blackshaw ${ }^{3}$, Ning Quan ${ }^{4,5}$ and Andy J. Fischer ${ }^{1 *}$ (D

\begin{abstract}
Background: Microglia and inflammation have context-specific impacts upon neuronal survival in different models of central nervous system (CNS) disease. Herein, we investigate how inflammatory mediators, including microglia, interleukin 1 beta (IL1 $\beta$ ), and signaling through interleukin 1 receptor type 1 (IL-1R1), influence the survival of retinal neurons in response to excitotoxic damage.

Methods: Excitotoxic retinal damage was induced via intraocular injections of NMDA. Microglial phenotype and neuronal survival were assessed by immunohistochemistry. Single-cell RNA sequencing was performed to obtain transcriptomic profiles. Microglia were ablated by using clodronate liposome or PLX5622. Retinas were treated with IL1 $\beta$ prior to NMDA damage and cell death was assessed in wild type, IL-1R1 null mice, and mice expressing IL-1R1 only in astrocytes.

Results: NMDA-induced damage included neuronal cell death, microglial reactivity, upregulation of pro-inflammatory cytokines, and genes associated with IL1 $\beta$-signaling in different types of retinal neurons and glia. Expression of the IL1 $\beta$ receptor, IL-1R1, was evident in astrocytes, endothelial cells, some Müller glia, and OFF bipolar cells. Ablation of microglia with clodronate liposomes or Csf1r antagonist (PLX5622) resulted in elevated cell death and diminished neuronal survival in excitotoxin-damaged retinas. Exogenous IL1 $\beta$ stimulated the proliferation and reactivity of microglia in the absence of damage, reduced numbers of dying cells in damaged retinas, and increased neuronal survival following an insult. IL1 $\beta$ failed to provide neuroprotection in the IL-1R1-null retina, but IL1 $\beta$-mediated neuroprotection was rescued when expression of IL-1R1 was restored in astrocytes.

Conclusions: We conclude that reactive microglia provide protection to retinal neurons, since the absence of microglia is detrimental to survival. We propose that, at least in part, the survival-influencing effects of microglia may be mediated by IL1 $\beta, I L-1 R 1$, and interactions of microglia and other macroglia.
\end{abstract}

Keywords: Microglia, IL1ß, IL-1R1, Retinal neuroprotection

\section{Background}

Microglia in the central nervous system have a significant impact upon neuronal function and survival following injury, and this impact can be beneficial or detrimental depending on the context [1]. Microglia are the innate immune cells of the central nervous system and are derived

\footnotetext{
*Correspondence: Andrew.Fischer@osumc.edu

${ }^{\dagger}$ Levi J. Todd and Isabella Palazzo contributed equally to this work.

'Department of Neuroscience, College of Medicine, The Ohio State

University, 3020 Graves Hall, 333 W. 10th Ave, Columbus, OH 43210-1239,

USA

Full list of author information is available at the end of the article
}

from erythro-myeloid precursor cells that originate in the yolk sac during early stages of development [2]. Prior to the onset of neurogenesis, microglia migrate into the retina through the vitreous and retinal periphery [3]. In the nervous system, microglia influence blood-vessel development, programmed cell death, phagocytosis of debris, and activity-dependent synaptic pruning $[4,5]$. In response to injury, microglia rapidly migrate to the site of injury and undergo morphological and molecular changes associated with inflammation $[1,6]$. Macrophages, another innate immune cell type, are often characterized by phenotypes that

(c) The Author(s). 2019 Open Access This article is distributed under the terms of the Creative Commons Attribution 4.0 International License (http://creativecommons.org/licenses/by/4.0/), which permits unrestricted use, distribution, and 
range across a spectrum; the "M1" activation profile is pro-inflammatory and cytotoxic, whereas the "M2" activation can promote tissue repair (reviewed by [7]). Microglia can also be activated in helpful and harmful manners (reviewed by [1]), although the existence of a simple polarized phenotype is doubtful $[8,9]$.

Upregulation of pro-inflammatory or neurotoxic molecules by activated microglia is widely considered to be deleterious to neuronal survival $[10,11]$. Multiple lines of evidence support this notion in diseased or damaged retinas. Activated microglia phagocytize healthy rod photoreceptors in a mouse model of retinitis pigmentosa and this exacerbates degeneration [12]. In a mouse model of glaucoma, upregulation of complement occurs prior to the onset of ganglion cell death, which tags synapses of ganglion cells to be targeted for engulfment by microglia [13]. In support of this finding, inhibition of complement suppresses the degeneration of RGCs in glaucomatous mouse retinas [14]. In a mouse model of hemorrhagic macular degeneration, treatment with minocycline prevents microglia accumulation in the sub-retinal space and increased photoreceptor survival [15]. However, in the context of retinal detachment, ablation of microglia prevented their accumulation in the sub-retinal space and this decreased survival of photoreceptors [16]. Multiple lines of evidence also support the notion that reactive microglia can be neuroprotective. In the chick retina, microglia ablation exacerbates NMDA-induced neuronal death [17]. Similarly, microglia are protective against excitotoxic damage in NMDA-treated hippocampal slice-cultures [18]. Ablation of microglia resulted in an increase in hippocampal neuron death in response to NMDA, and replenishment of microglia restored the resistance of hippocampal neurons to excitotoxicity [18]. In some paradigms, microglia can support neuronal survival during injury, but become detrimental to survival during the recovery phase [19]. Considered together, microglia can be beneficial or harmful to neuronal survival and the survival-influencing actions of microglia are context-specific.

In this study, we investigate whether microglia influence neuronal survival following an excitotoxic insult in the mouse retina. In addition, we investigate the involvement of IL1 $\beta$ and cell type-specific IL-1R1-receptor signaling. Collectively, our findings suggest that reactive microglia provide neuroprotection in excitotoxin-damaged retinas and this may be mediated by microglial production of IL1 $\beta$, which acts, in part, via IL-1R1 in macroglia.

\section{Methods and materials}

\section{Animals}

The use of animals in these experiments was in accordance with the guidelines established by the National Institutes of Health and the Ohio State University. Mice were kept on a cycle of $12 \mathrm{~h}$ light, $12 \mathrm{~h}$ dark (lights on at
6:00 AM). C57BL/6J mice between the ages of P40-P100 were used for all experiments except when noted. Lines of mice included IL-1R1 reporter mice (IL-1R1GR/GR), IL-1R1 null mice (IL-1R1-r/r), and GFAPCre-IL-1R1r/r [20]. GFAPCre-IL-1R1r/r mice are made from GFAP $>$ Cre $x$ homozygous knock in stop-fl/fl-IL1R1-3HA-IRES-tdTomato line of mice that was crossed onto the IL-1R1-null background [20, 21].

\section{Preparation of clodronate liposomes}

The preparation of clodronate liposomes was similar to previous descriptions [22, 23]. Fifty nanograms of cholesterol and $8 \mathrm{mg}$ egg lecithin were dissolved in chloroform in a round-bottom flask. The solution was evaporated until a white liposome residue remained. One hundred and fifty-eight milligrams of dichloro-methylene diphosphonate (clodronate) in sterile PBS was added and rotated for $10 \mathrm{~min}$. The liposomes were sealed under $\mathrm{N}_{2}$ at room temperature for $2 \mathrm{~h}$. Clodronate encapsulation was facilitated by sonication for $3 \mathrm{~min}$. The liposomes were centrifuged at $10,000 \times g$ for $15 \mathrm{~min}$ and re-suspended in $150 \mathrm{ml}$ PBS. We are unable to determine the clodronate concentration due to the stochastic nature of the clodronate combining with the liposomes. We tittered doses to levels where $>70 \%$ of the microglia were ablated at 1 day after treatment.

\section{Oral administration of PLX5622}

C57BL/6 mice were fed chow formulated with PLX5622 (1200 ppm; provided by Plexxikon). Control animals were fed control chow AIN-76A (provided by Plexxikon). Mice were fed ad libitum on PLX5622 or control diets for a minimum of 2 weeks before experiments, and this diet was continued through the duration of each experiment.

\section{Intraocular injections}

Mice were anesthetized by using an isoflurane/oxygen non-rebreathing inhaler; $98 \%$ oxygen and $2 \%$ isoflurane. Injections were made into the vitreous chamber of the eye through the dorsal sclera. Injections are made by using a 20- $\mu$ l Hamilton syringe with a disposable custom 31-gauge needle with a cutting tip. The volume of all injections was 2-3 $\mu \mathrm{l}$. For all experiments, the right eyes of mice were injected with the "test" compound and the contra-lateral left eyes were injected with vehicle as a control. Compounds were injected in $2 \mu \mathrm{l}$ sterile saline. Compounds used in these studies included $N$-methyl-D-aspartate (NMDA; 38.5 or $154 \mu \mathrm{g}$ / dose) and IL1 $\beta$ (200 ng/dose; R\&D systems).

\section{scRNA-seq}

Retinas were acutely dissociated via papain digestion and mild trituration. Dissociated cells were loaded onto the 
10X Chromium Controller using Chromium Single Cell 3' v2 reagents. Sequencing libraries were prepared following the manufacturer's instructions (10X Genomics), with 10 cycles used for cDNA amplification and $12 \mathrm{cy}$ cles for library amplification. The resulting sequencing libraries were sequenced with paired end reads, with Read 1 (26 base pairs) and Read 2 (98 base pairs), on anNextseq500 at the Genomics Resources Core Facility (High Throughput Center) at Johns Hopkins University. Raw sequence data was processed with Cell Ranger software (10X Genomics) to align sequences, de-multiplex, and annotated to ENSMBL databases; count reads; assess levels of expression; and construct gene-cell matrices. $t$-Distributed Stochastic Neighbor Embedding (tSNE) plots were generated and probed using Cell Ranger and Cell Browser software (10X Genomics). The tSNE plots were generated via aggregate cluster analysis of 9 separate cDNA libraries, including 2 replicates of control undamaged retinas and retinas at different times after NMDAtreatment. The identity of clustered cells was established using known cell type-specific markers. Violin/scatter plots were generated using Seurat [24, 25]. Identification of cell types clustered together in tSNE plots was established by using a candidate approach using well-known cell type-specific markers, as follows: ganglion cells (Pou4f2, Thy1, Nefl), amacrine cells (Tfap2a, Pax6, Gad1), bipolar cells (Vsx2, Grm6, Grik1), horizontal cells (Calb1, Lhx1), rod photoreceptors (Rho, Nrl, Nr2e3), cone photoreceptors (Opn1mw, Arr3), astrocytes (Pax2, S100b), Müller glia (Vim, Slc3a2, Glul, Rlbp1), pericytes (Tagln, Acta2), and endothelial cells (Tie1, Cldn5).

\section{Fixation, sectioning, and immunocytochemistry}

Tissues were fixed, sectioned, and immunolabeled as described previously [26-28]. None of the observed labeling was due to non-specific labeling of secondary antibodies or auto-fluorescence because sections labeled with secondary antibodies alone were devoid of fluorescence. Primary antibodies used in this study are described in Table 1. Secondary antibodies included donkey-anti-goatAlexa488/568, goat-anti-rabbit-Alexa488/568, and goat-antimouse-Alexa488/568/647 (Thermo Fisher Scientific) diluted to $1: 1000$ in PBS plus $0.2 \%$ Triton X-100.

\section{Terminal deoxynucleotidyl transferase dUTP nick end labeling (TUNEL)}

To identify dying cells that contained fragmented DNA, the TUNEL method was used. We used an In Situ Cell Death Kit (TMR red; Roche Applied Science), as per the manufacturer's instructions.

\section{Photography, measurements, cell counts, and statistics} Photomicrographs were obtained using a Leica DM5000B microscope equipped with epifluorescence and Leica DC500 digital camera. Confocal images were obtained using a Leica SP8 imaging system at the Department of Neuroscience Imaging Facility at the Ohio State University. Images were optimized for color, brightness, and contrast, multiple channels over-laid, and figures constructed by using Adobe Photoshop. Cell counts were performed on representative images. Counts were consistently made from central regions of retina that were within a $0.7-\mathrm{mm}$ radius of the posterior pole of the eye.

Where significance of difference was determined between two treatment groups accounting for inter-individual variability (means of treated-control values), we performed a two-tailed, paired $t$ test. Where significance of difference was determined between two treatment groups, we performed a two-tailed, unpaired $t$ test. Where significance of difference was determined across multiple groups, we performed a one-way ANOVA, followed by Tukey's test for multiple comparisons.

Table 1 Antibodies, sources, and working dilutions

\begin{tabular}{lllll}
\hline Antigen & Working dilution & Host & Clone or catalog number & Source \\
\hline Calretinin & $1: 1000$ & Rabbit & CR 7697 & Swant Inc. \\
Draq5 & $1: 2000$ & n/a & 62251 & Thermo Scientific \\
F4/80 & $1: 250$ & Mouse & MCA497GA & Bio-Rad Laboratories \\
Iba1 & $1: 1000$ & Rabbit & $019-19741$ & Wako Pure Chemical Industries \\
Ki67 & $1: 300$ & Rabbit & DRM004 & OriGene \\
Pax6 & Rabbit & $50-103-0098$ & Biolegend \\
Tomato Lectin & $1: 1000$ & n/a & B-1175 & Vector Laboratories \\
GFP & $1: 500$ & Chicken & Ab13970 & Abcam \\
CD31 & $1: 1000$ & Mouse & 102502 & Biolegend \\
RFP & $1: 500$ & Goat & Ab8181-200 & Origene Technologies \\
S100b & Rabbit & Ab227914 & Abcam \\
\hline
\end{tabular}




\section{Results}

Microglia reactivity accompanies NMDA-induced cell death

Reactive microglia are known to be prevalent in damaged retinas [4]. We characterized the progression of reactive microglia and cell death in the retina following a single intravitreal injection of NMDA. Consistent with previous reports [29], we found that Iba1-postive microglia exhibited an amoeboid "reactive" morphology shortly after NMDA-damage. This morphology was prevalent among microglia at 24 and $48 \mathrm{~h}$ after treatment, and there was a significant increase in the total number of Iba1-positive microglia at $4 \mathrm{~h}, 24 \mathrm{~h}$, and $48 \mathrm{~h}$ after NMDA (Fig. 1a, b). TUNEL-positive cells were detected as early as $4 \mathrm{~h}$ after NMDA-treatment (Fig. 1c, d). The abundance of dying cells peaked at $24 \mathrm{~h}$ and was diminished by $48 \mathrm{~h}$ after treatment (Fig. 1c, d). At $48 \mathrm{~h}$ after damage, we observed a significant increase in the number of Iba1-positive cells that expressed F4/80 (Fig. 1e, f); a marker that is upregulated by reactive microglia [30, 31]. TSPO has been reported as a marker for reactive microglia and has been implicated with the ligand Dbi as coordinating pro-inflammatory signals between microglia and Müller glia in the retina [32, 33]. However, we found that TSPO is upregulated in both microglia and Müller glia, whereas $D b i$ is upregulated in microglia and downregulated in Müller glia in NMDA-damaged retinas (Additional file 1: Figure S1), consistent with a recent report [34]. The antibodies to Iba1 and F4/80 do not distinguish between microglia and macrophage that may have migrated into the retina. Thus, when describing Iba1/F4/ 80-positive microglia, it is implied that these cells may include macrophages.

\section{The ablation of microglia exacerbates cell death in damaged retinas}

We investigated whether the ablation of microglia/macrophages in the retina influenced the survival of retinal neurons after an excitotoxic insult. Two consecutive daily intraocular injections of clodronate-liposomes depleted nearly $70 \%$ of the Iba1-positive microglia/macrophages in the retina (Fig. 2a, b). Empty liposomes were not used as a control because the liposomes potently stimulate the reactivity of microglia [22]. We did not detect any dying, TUNEL-positive cells in undamaged retinas following treatment with clodronate-liposome (not shown), suggesting that the clodronate-liposomes do not directly influence survival of retinal neurons or macroglia and that the destruction of the microglia is rapid and/or does not involve the fragmentation of DNA. With the majority of the microglia missing from the retina, we found more than a twofold increase in the number of TUNEL-positive cells in the retina after NMDA-treatment (Fig. 2c, d). The increase in cell death corresponded with decreased numbers of Pax6- and calretinin-positive cells in the ganglion cell layer (GCL) at 10 days after NMDA-treatment (Fig. 2e, f).

It is possible that the few remaining microglia that evade destruction with the clodronate-liposomes exacerbate the neuronal death resulting from NMDA-treatment. Thus, as an alternative means of ablating retinal microglia, we treated mice with chronic exposure to Csf1r antagonist (PLX5622), which selectively ablates microglia in the CNS [35]. Consistent with previous reports [36], we found a near-complete ablation of microglia from the retina after 2 weeks of PLX5622-treatment (Fig. 3a). In retinas treated with PLX5622 and NMDA, we found that less than 1\% of the Iba1-positive cells survive exposure to PLX5622 (Fig. $3 \mathrm{~d})$. With the ablation of nearly all microglia at the time of injury, we found more than a twofold increase in the number of TUNEL-positive cells in the inner nuclear layer (INL) and GCL after NMDA-damage (Fig. 3b, c). Consistent with our findings in clodronate-treated retinas, there was a decrease in numbers of Pax6- and calretinin-positive neurons at 10 days after NMDA-damage in PLX5622treated retinas (Fig. 3d-f).

\section{Single-cell RNA-seq; expression of IL1 $\beta$ signaling components in the retina}

To identify and provide a context for the IL-1-related factors that may mediate signaling in the different retinal cell types, we performed single-cell RNA sequencing (scRNA-seq) on NMDA-damaged mouse retina (Fig. 4). tSNE plots were generated from aggregate sequence data, 9 different single-cell cDNA libraries, including two preparations (rep1 and rep2) from undamaged retinas, and cells from retinas at 3, 6, 12, 24, 36, 48, and 72 $\mathrm{h}$ after NMDA-treatment (Fig. 4a). tSNE plots revealed distinct clustering of different retinal cell-types (Fig. 4a, b). The Müller glia were identified based on distinct and elevated expression, compared to all other cell types, of Vim, Lhx2, Sox9, Rlbp1, and Slc1a3 (Fig. 4c). Relatively few astrocytes (143 cells) were identified, based on expression of $5100 \beta$ and $P a x 2$, (not shown) and were clustered in close proximity to the Müller glia. The small number of astrocytes reflects the relatively low abundance of astrocytes among retinal cell types. However, we cannot exclude the possibility of capture-bias wherein cell dissociation methodologies and micro-fluidic capture impacts the chances of capturing particular cell types. The clusters of bipolar cells were identified based on combined expression of Vsx2, Otx2, Lhx3, Grm6, and Grik1 (not shown). The cluster of endothelial cells was identified based on combined, distinct expression of Tie1 and Cldn5 (not shown). Microglia were identified based on combined, distinct expression of Rgs1, Ccl3, Ccl6, C1qa, C1qb, C1qc, Trem2, and Csf1r (Fig. 4d, e). The pattern of expression of Csf1r is consistent with the specific actions of PLX5622 at this receptor and selective ablation of microglia; Csflr was 

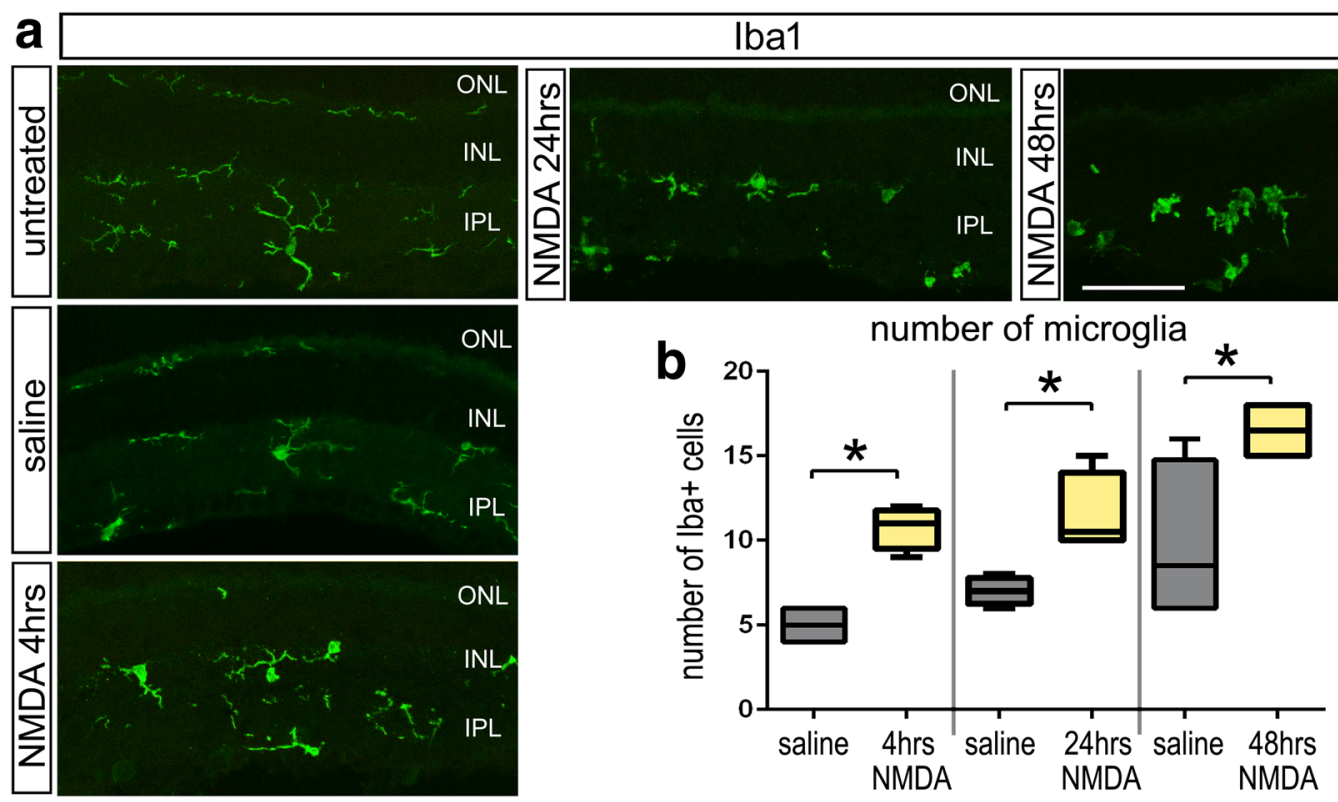

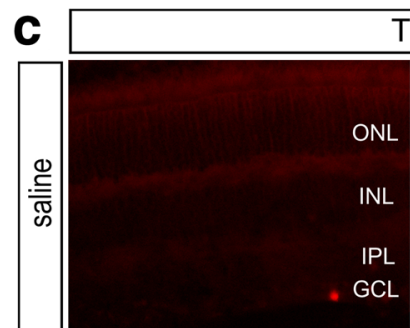

\section{TUNEL}

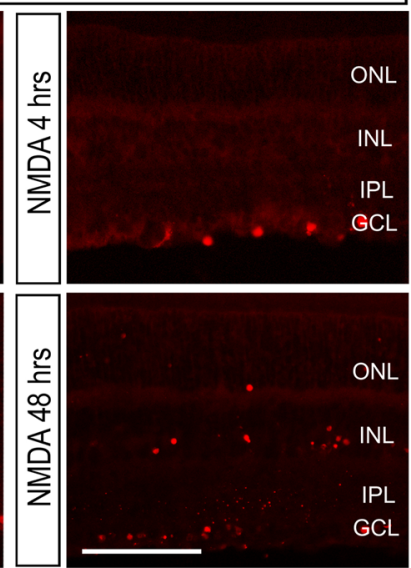

e
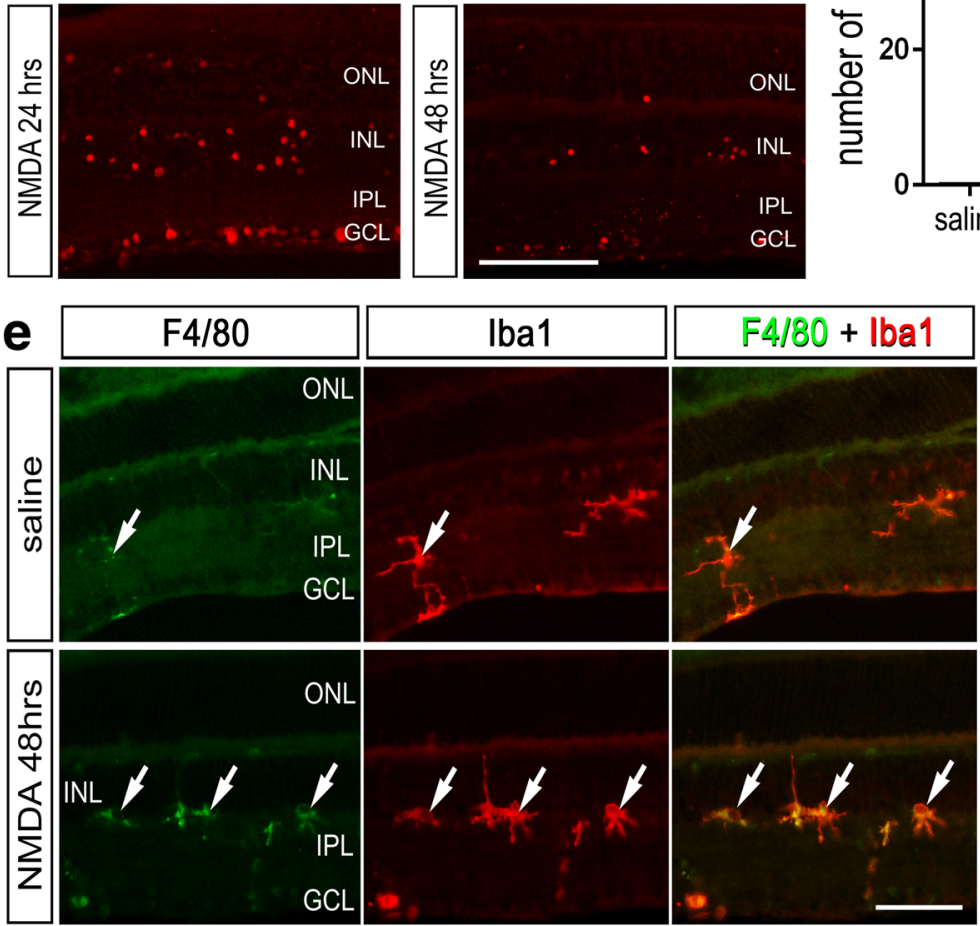
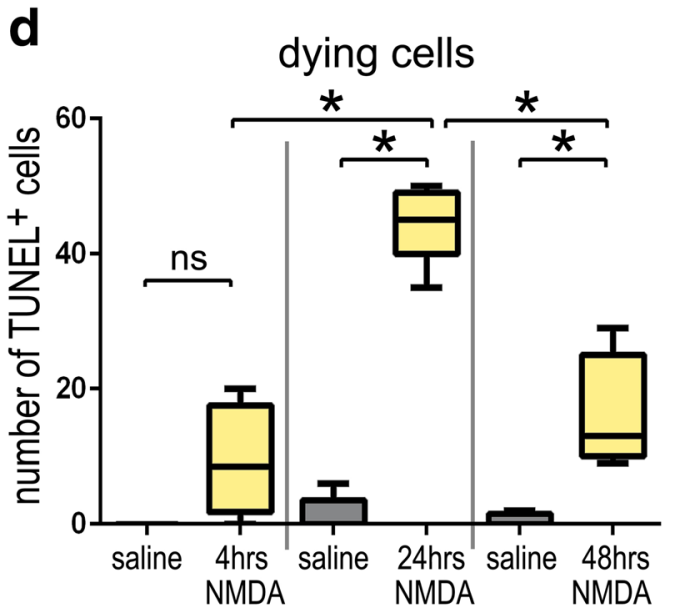
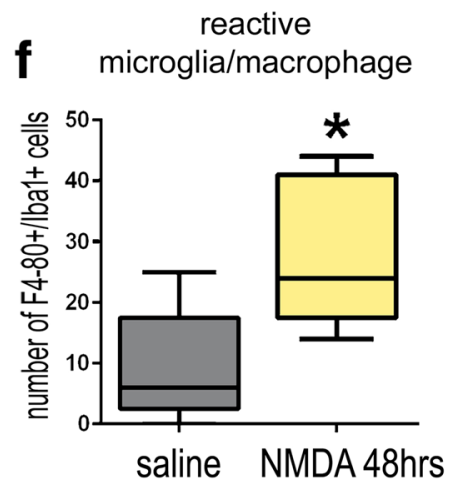

Fig. 1 (See legend on next page.) 
(See figure on previous page.)

Fig. 1 Reactive microglia accumulate in NMDA-damaged retinas. Eyes were injected with a single dose of NMDA and retinas harvested at different times after treatment. Sections were labeled with antibodies to Iba1 (a) and TUNEL (c), or antibodies to F4/80 (green) and Iba1 (red; e).

The boxplots in $\mathbf{b}$ illustrate the mean, upper extreme, lower extreme, upper quartile, and lower quartile for total number of microglia in the retina at different times after NMDA-treatment $(n=4$ animals).The boxplots in $\mathbf{d}$ illustrate the mean, upper extreme, lower extreme, upper quartile, and lower quartile for number of TUNEL-positive cells in the retina at different times after NMDA-treatment ( $n=4$ animals). The box plot in $\mathbf{f}$ illustrates the mean, upper extreme, lower extreme, upper quartile, and lower quartile for number of F4/80-positive/lba1-positive cells ( $n \geq 6$ animals). Significance of difference $\left({ }^{*} p<0.05\right)$ was determined by using a $t$ test $(\mathbf{e})$. Arrow-heads indicate microglia. The calibration bar panels $\mathbf{a}$, c, and e represent $50 \mu \mathrm{m}$. Abbreviations: ONL, outer nuclear layer; INL, inner nuclear layer; IPL, inner plexiform layer; GCL, ganglion cell layer

not detected in significant numbers in retinal neurons, astrocytes, endothelial cells, or retinal pigmented epithelial (RPE) cells (Fig. 4e), suggesting that the diminished neuronal survival observed in PLX5622-treated animals resulted from specific actions at Csf1r expressed by microglia in the retina. Very few microglia from control, undamaged retinas were surveyed by scRNA-seq; less than $5 \%$ of the total number of microglia sampled came from control retinas whereas $34.4 \%$ of the total number of cells in the aggregate came from control retinas. Microglia from damaged retinas make up $95 \%$ of the total number of microglia captured. These findings suggest that quiescent microglia do not tolerate the dissociation and capture process for scRNA-seq as well as microglia from damaged retinas.

IL-1 signaling plays a prominent role in inflammatory responses [37]. Interleukin-1 $\alpha$ (IL1 $\alpha)$ and interleukin-1 $\beta$ (IL1 $\beta$ ) both bind to IL-1R1 to initiate cell signaling. We queried for expression of cytokines, IL1-receptors, and associated signal transduction genes (Fig. 4f). We found that the secreted ligands Tnfo, Ill $\beta$, and Il1 $\alpha$ were expressed exclusively by microglia, particularly following NMDA-treatment (Fig. 4g). Tnf $\alpha, I l 1 \beta$, and $I l 1 \alpha$ were rapidly upregulated within microglia at $3 \mathrm{~h}$ after NMDAtreatment, and levels of expression remained elevated at 6 and $12 \mathrm{~h}$ after treatment but were reduced thereafter (Fig. 4h).Tnfrsfria is a predominant receptor for TNF $\alpha$ [38, 39]. Tnfrsfria expression was detected in endothelial cells, pericytes, astrocytes, and Müller glia in NMDA-damaged retinas (Fig. 4i, j). Tnfsfr $12 a$ was highly expressed by Müller glia at 3, 6, $12+24$, and $36+48+72 \mathrm{~h}$ after NMDA-treatment and expressed in scattered microglia (Additional file 2: Figure S2). Tnfrsfr21 was expressed at relatively high levels in scattered microglia and Müller glia in control and damaged retinas (Additional file 2: Figure S2). Tnfrsfr $1 b$, Tnfrsfr $11 a$, and Tnfrsfr $13 b$ were expressed at relatively high levels in scattered microglia, but not other types of retinal cells (Additional file 2: Figure S2). Other isoforms of the Tnf-receptor (Tnfrsfr4, 8, 9, 10a, $11 b, 13 c, 17,18,19,22,23,25,26)$ were not expressed at appreciable levels in retinal cells (not shown). By contrast, a recent study implied that retinal ganglion cells express receptors for TNF $\alpha$, which is inconsistent with our findings [40]. By comparison, the receptor $I l 1 r 1$ was predominantly expressed by astrocytes, and scattered expression was observed in endothelial cells, OFF bipolar cells, and some Müller glia (Fig. 4k, l). We failed to detect Il1r1 in microglia (Fig. 4k, l), suggesting that the reactivity and proliferation of microglia that resulted from exogenous IL1 $\beta$ was an indirect effect. We detected low levels of Illr2 in only 5/655 microglia in the retina, consistent with RNA-seq data of purified cell types from the mouse CNS wherein Il1r2 was detected at very low levels in microglia/ macrophage [10, 41]. Additionally, scRNA-seq from primate retina failed to detect appreciable levels of Il1r2 [42]. Myd88 serves as the canonical signaling adaptor for the IL-1 pathway and functions by linking IL-1R or TLRs to kinases (IRAK 1,2, and 4) [43, 44]. Activation of IRAKs can lead to downstream activation of the NFKB pathway [45]. These IL-1R1-associated signal-transduction genes were expressed in both microglia and Müller glia from NMDA-damaged retinas (Fig. 4f). The signal-transduction components are likely involved in TLR-signaling. Thus, we probed for changes in TLR1-13 after damage. We found scattered expression of Tlr2 and Tlr3 in Müller glia and scattered expression of Tlr1, Tlr2, Tlr3, Tlr4, Tlr7, and Tlr13 in microglia (Additional file 3: Figure S3). These findings are consistent with the hypothesis that microglia do not directly respond to Il1 $\beta$.

To corroborate the data from scRNA-seq, we probed for the expression of IL-1R1 by using an IL-1R13HA-IRES-tdTomato knock-in mouse line in which an IRES-tdTomato sequence is inserted in the $3^{\prime}$ end of Il-1r1 mRNA [20]. Consistent with our scRNA-seq data, immunohistochemistry labeling of RFP, which detects tdTomato, appeared in bipolar cells, astrocytes, endothelial cells, and a few Müller glia (Fig. 5a). Patterns of fluorescence did not appear different between control, NMDA-treated, and IL1 $\beta$-treated retinas, and the expression of IL-1R1 did not vary significantly among individual cell types (Fig. 5a-e). IL-1R1-RFP was observed in nearly all endothelial cells that were CD31-positive and nearly all astrocytes that were Sox9- or S100 $\beta$-positive (Fig. 5b, c, e). IL-1R1-RFP was detected in 4-10\% of Müller glia that were positive for Sox9, which specifically labels Müller glia in the INL (Fig. 5c-e). IL-1R1-RFP-positive bipolar cells were positive for Vsx2 and Otx2 (Fig. $5 \mathrm{c}-\mathrm{e})$; these cells were presumptive OFF bipolar cells, consistent with the scRNA-seq data. Between 10 and 


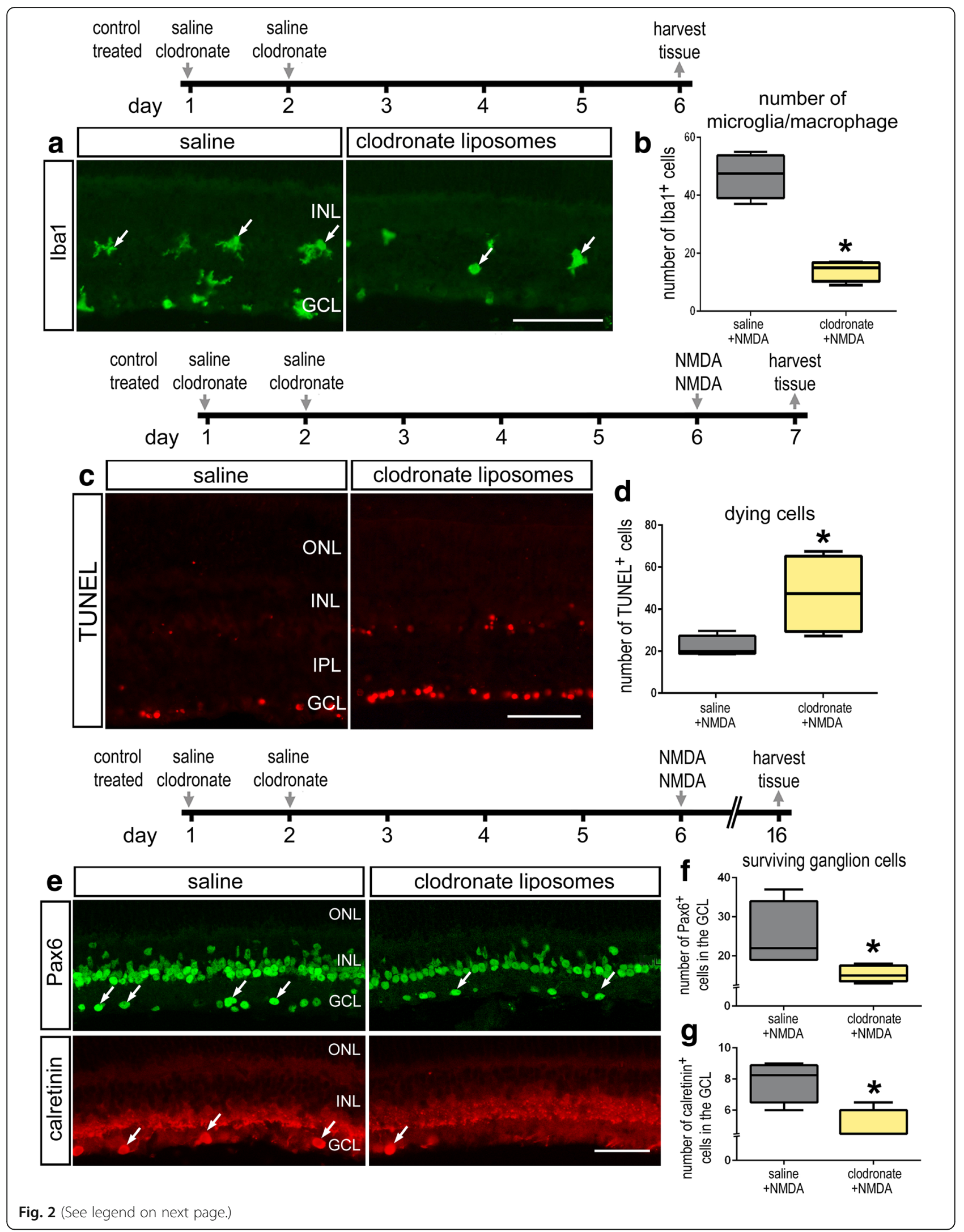


(See figure on previous page.)

Fig. 2 Clodronate-liposome-mediated ablation of microglia results in increased cell death and diminished neuronal survival in damaged retinas. Eyes were treated with 2 consecutive daily injections of saline or clodronate-liposomes and tissue harvested 4 days later (a, $\mathbf{b})$, injected with NMDA at 4 days after clodronate-liposomes and harvested 1 day later $(\mathbf{c}, \mathbf{d})$, or injected with NMDA at 4 days after clodronate-liposomes and harvested 10 days later (e-g). Sections of the retina were labeled with antibodies to Iba1 (a), TUNEL (c), antibodies to Pax6 (green; e), or calretinin (red; e). The box plots in $\mathbf{b}, \mathbf{d}, \mathbf{f}$, and $\mathbf{g}$ illustrate the mean, upper extreme, lower extreme, upper quartile, and lower quartile ( $n \geq 6$ animals). Significance of difference $\left(^{*} p<\right.$ 0.05) was determined by using a $t$ test. Arrows indicate microglia (a) or surviving ganglion cells (e). The calibration bars panels $\mathbf{a}$, $\mathbf{c}$, and $\mathbf{e}$ represent 50 mm. Abbreviations: ONL, outer nuclear layer; INL, inner nuclear layer; IPL, inner plexiform layer; GCL, ganglion cell layer

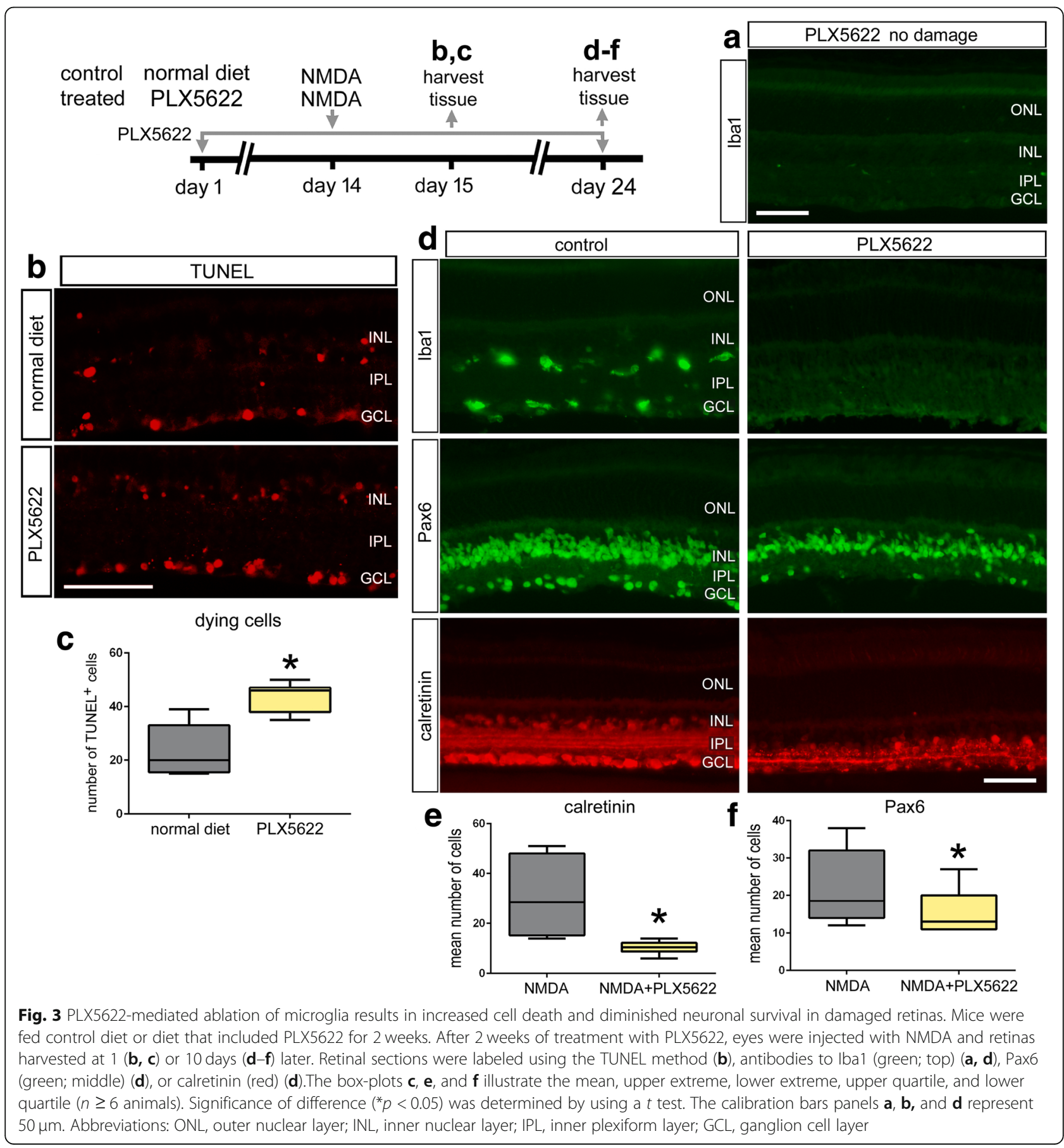




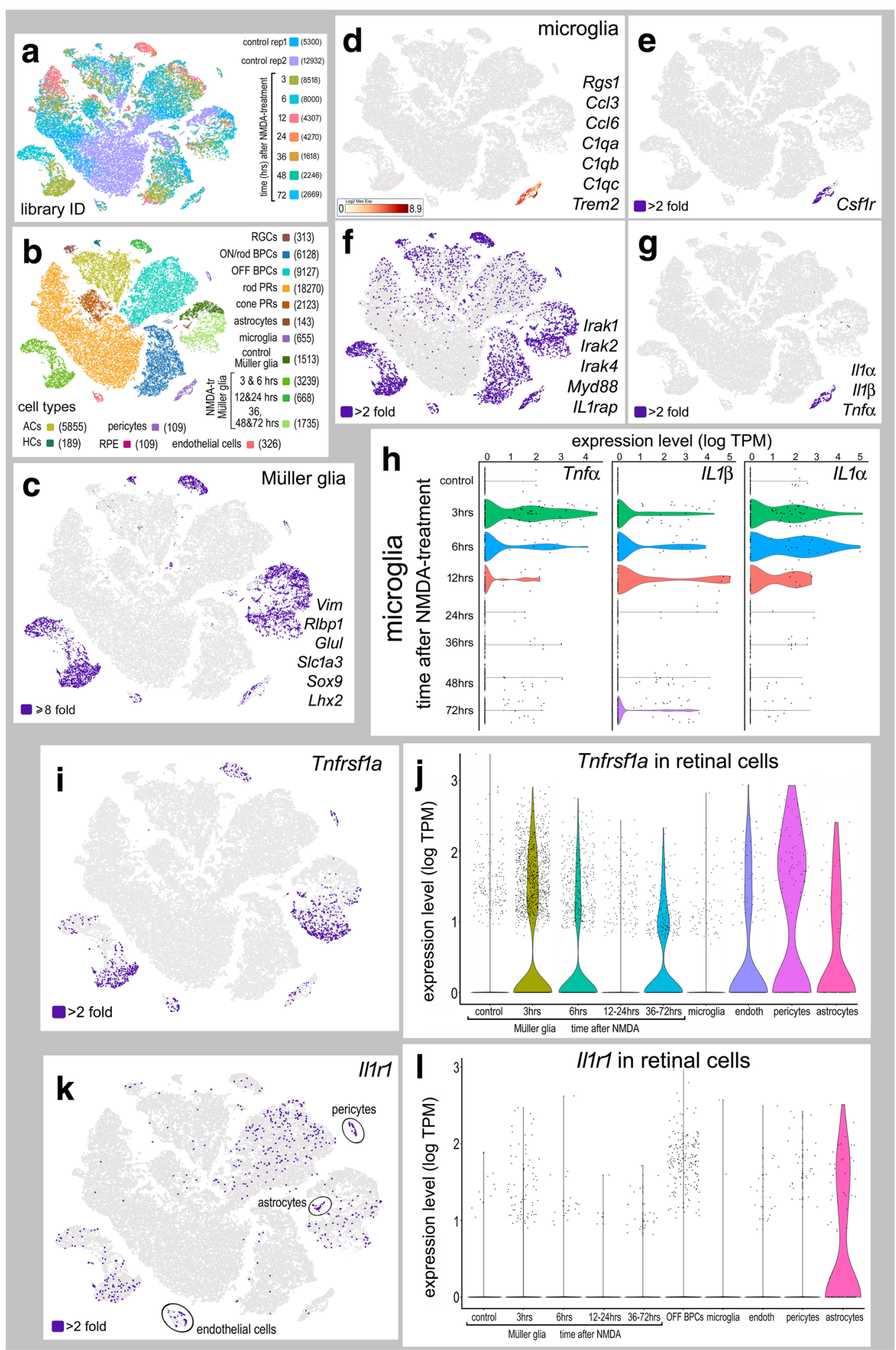

Fig. 4 (See legend on next page.) 
(See figure on previous page.)

Fig. 4 Expression of inflammatory cytokines and IL1-receptor-related genes in retinal cells following NMDA-treatment. scRNA-seq was used to identify patterns of expression of IL1 $\beta$-related genes among acutely dissociated retinal cells. Each dot represents one cell. Cells were sampled from control retinas (rep1 5300 cells and rep2 12,932 cells), and from retinas at $3 \mathrm{~h}$ (8518 cells), $6 \mathrm{~h}$ (8000 cells), $12 \mathrm{~h}$ (4307 cells), $24 \mathrm{~h}$ (4270 cells), $36 \mathrm{~h}$ (1618 cells), $48 \mathrm{~h}$ (2246 cells), and $72 \mathrm{~h}$ (2269 cells) after NMDA-treatment (a). tSNE plots revealed distinct clustering of different types of retinal cells and numbers of cells surveyed (in parentheses) (b). Müller glia were identified based on significant ( $\geq 8$-fold; purple dots) collective expression of Lhx2, Sox9, Rlbp1, or Slcla3 (c). Microglia were identified based on collective expression of Rgs1, Trem2, CCl3, CCl4, Claa, Clab, and C1qc (d). e t-SNE plot for expression ( $\geq 2$-fold; purple dots) of Csf1r, the target of PLX5622, which was detected only in microglia. $\mathbf{f}$ t-SNE plot for the collective expression ( $\geq 2$-fold; purple dots) of components of IL1-receptor signaling, Irak1, Irak2, Irak4, IL Irap, and Myd88. g t-SNE plot for the collective expression of $I L 1 a, I L 1 \beta$, and TNFa. Violin/scatter plots of $I L 1 a, I L 1 \beta$, and TNFa in microglia at different times after NMDA-treatment (h). $\mathbf{i}$ t-SNE plot for expression ( $\geq 2$-fold) of Tnfffr1a in endothelial cells, astrocytes, OFF bipolar cells, and Müller glia. $\mathbf{j}$ Violin/scatter plots of Tnfsfr1a in microglia, endothelial cells, pericytes, astrocytes, and Müller glia at different times after NMDA-treatment. $\mathbf{k}$ t-SNE plot for expression ( $\geq 2$-fold) of $\| 1 / r 1$ in endothelial cells, astrocytes, OFF bipolar cells, and Müller glia. I Violin/scatter plots of $/ 11 r 1$ in microglia, endothelial cells, pericytes, astrocytes, and Müller glia at different times after NMDA-treatment

$20 \%$ of the bipolar cells and 4 and $10 \%$ of the Müller glia were positive for RFP regardless of treatment (Fig. 5e). We validated patterns of RFP expression, identifying Il-1r1 mRNA expression, by labeling for HA, which is tagged to the carboxy terminal of IL-1R1. HA-labeling is consistent with the patterns of expression seen with RFP with labeling predominant in astrocytes (Additional file 4: Figure S4).

\section{Exogenous IL1 $\beta$ activates microglia and conveys neuroprotection}

We next sought to examine the effect of the pro-inflammatory cytokine IL1 $\beta$ on retinal microglia. Compared to the microglia observed in saline-treated retinas, IL1 $\beta$-treatment resulted in a significant increase in both the total number of microglia and F4/80-positive cells (Fig. $6 \mathrm{a}-\mathrm{c}$ ). IL1 $\beta$ stimulated the proliferation of microglia; we found a significant increase in the number of Ki67/tomato-lectin-positive cells in treated retinas compared to numbers seen in control retinas (Fig. 6d, e). There were no TUNEL-positive cells detected in IL1 $\beta$ treated retinas, suggesting that cell death was not induced. These results suggest that a single intravitreal injection of IL1 $\beta$ is sufficient to stimulate microglial reactivity in the absence of damage, and this effect is likely to be indirect because we failed to detect significant expression of IL-1R1 in microglia via scRNA-seq or IL-1R1-reporter (Fig. 4k, l and 5).

Reactive microglia can influence the survival of neurons in the CNS, including the retina (reviewed by [4, 46-48]). Thus, we investigated whether IL1 $\beta$-mediated activation of microglia influenced the survival of neurons in NMDA-damaged retinas. We found that a single intraocular injection of IL1 $\beta$ prior to NMDA-treatment resulted in a significant decrease in the number of TUNEL-positive cells at 1 day after damage (Fig. 6f, g). Consistent with these findings, we found significant increases in the number of Draq5-labeled nuclei in the GCL, calretinin-positive ganglion cells in the GCL, and thickness of the inner nuclear layer (INL) when assayed
12 days after damage (Fig. 6h-k). In addition, we observed increased numbers of reactive microglia labeled for Iba1 and F4/80 in IL1 $\beta$-treated retinas (Fig. 6l, m). Collectively, these findings suggest that intravitreal delivery of IL1 $\beta$ stimulates the activation of microglia and protects inner retinal neurons and ganglion cells against excitotoxic damage.

\section{IL1 $\beta$ protects neurons from excitotoxic damage independent of microglia}

We next sought to test whether the neuroprotective effects of IL1 $\beta$ were mediated through microglia/macrophage in the retina. We found that IL1 $\beta$ application was protective against cell death at $24 \mathrm{~h}$ post injury even with depletion of microglia (Fig. 7a, b). Although we found increased numbers of microglia/macrophage in NMDAdamaged retinas that were pre-treated with IL1 $\beta$ compared to damaged retinas alone (Fig. 6), there was a near complete depletion of microglia in IL1 $3 /$ NMDA-treated retinas from mice fed a diet containing PLX5622 (Fig. 7c, d). All regions of retinas from mice treated with PLX5622, IL1 $\beta$, and NMDA were depleted of Iba1-positive cells (Fig. 7c, d). However, Iba1-positive cells were observed within the optic nerve near the optic nerve head of PLX5622treated mice following damage (Fig. 7e). Iba1-positive cells were present after NMDA damage with or without IL1 $\beta$ pre-treatment (not shown). These findings suggest that IL1 $\beta$ treatment conveys neuroprotection against excitotoxic damage independent of the presence of microglia, and the microglia/monocytes within the optic nerve survive exposure to PLX5622.

\section{IL1 $\beta$ conveys neuroprotection by acting through IL-1R1 expressed by astrocytes}

Considered together, our data suggest that IL-1R1-signaling may originate with pro-inflammatory cytokines provided by microglia/macrophage and broadly impact retinal neurons (namely OFF bipolar cells), endothelial cells, astrocytes, and Müller glia in damaged retinas. To further explore the involvement of IL-1R1-signaling, we 


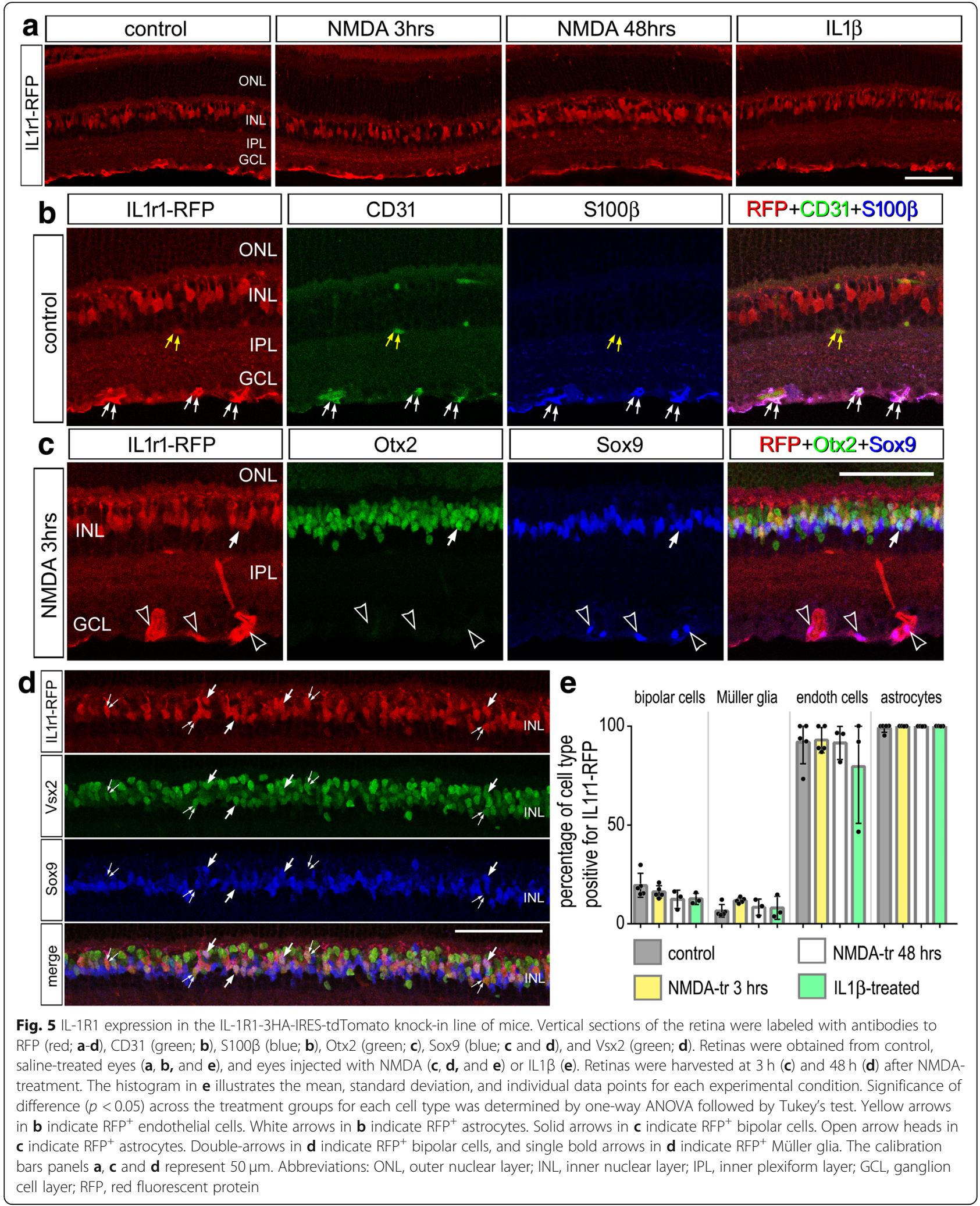

investigated the impact of NMDA-induced damage in mutant retinas of $\mathrm{IL}-1 \mathrm{R} 1 \mathrm{r} / \mathrm{r}$ mice (IL-1R1-null) and GFAPCre-IL-1R1r/r mice (IL-1R1 expressed only in
astrocytes).The GFAP-Cre-IL-1R1 mice are made from GFAP-Cre $x$ homozygous knock in stop-fl/fl-IL-1R1-3HA-IRES-tdTomato line of mice that was crossed onto 


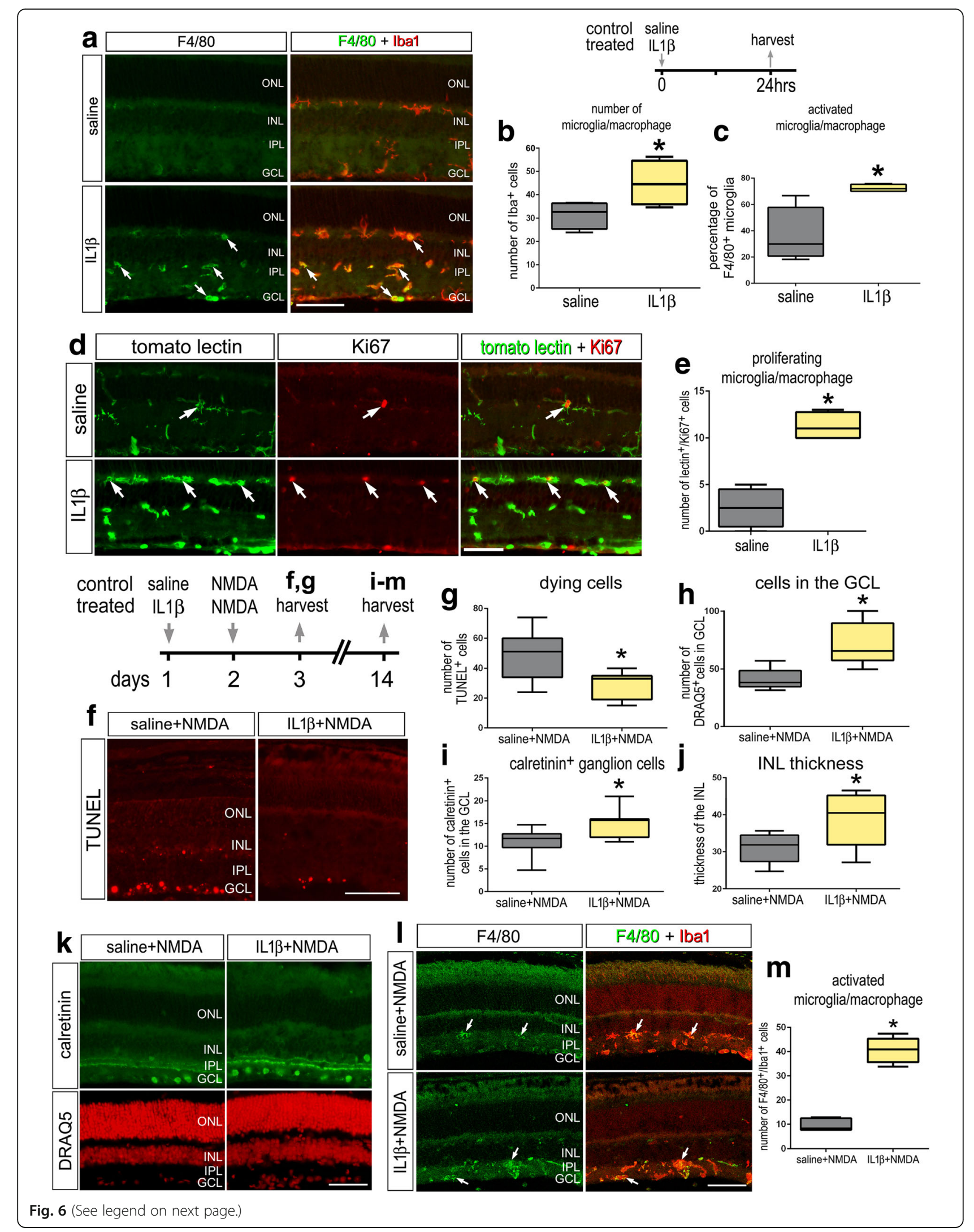


(See figure on previous page.)

Fig. 6 IL1 $\beta$ stimulates the reactivity and proliferation of microglia in the retina and conveys neuroprotection following NMDA-treatment. Eyes were injected with saline or IL $1 \beta$, and retinas harvested $24 \mathrm{~h}$ later (a-e). Eyes were injected with saline or IL $1 \beta$ on day 1 , NMDA on day 2, and retinas harvested 1 or 12 days later (f-m). Retinal sections were labeled with TUNEL (f) or DRAQ5 (red; $\mathbf{k}$ ) or antibodies to F4/80 (green) and Iba1 (red; a), tomato lectin (green; $\mathbf{d}$ ) and Ki67 (red; $\mathbf{d}$ ), calretinin (green; k), or F4/80 (green) and Iba1 (red; I). The box plots in b, c, e, $\mathbf{g}-\mathbf{j}$, and $\mathbf{m}$ illustrate the mean, upper extreme, lower extreme, upper quartile, and lower quartile ( $n \geq 6$ animals). Significance of difference $\left(^{*} p<0.05\right)$ was determined by using a $t$ test. Arrows indicate microglia. The calibration bars in panels $\mathbf{a}, \mathbf{d}, \mathbf{f}, \mathbf{k}$, and $\mathbf{I}$ represents $50 \mu \mathrm{m}$. Abbreviations: ONL, outer nuclear layer; INL, inner nuclear layer; IPL, inner plexiform layer; $G C L$, ganglion cell layer

the IL-1R1-null background [20, 21]. With this partial GFAP-promoter, IL-1R1 is restored only in astrocytes, not in Müller glia, according to the IRES-reporter; the tdTomato-reporter was detected exclusively in S100 $\beta$-positive astrocytes (Fig. 8e). Additionally, HA-labeling revealed that IL-1R1 was specifically restored in astrocytes near the vitread surface of the retinas (Additional file 4: Figure S4). We found that levels of cell death following NMDA-treatment are more variable but not significantly different in IL-1R1-null retinas compared to wild type (WT) retinas (Fig. $8 \mathrm{a}-\mathrm{d}$ ). The germline-loss of IL-1R1 may result in compensatory adjustments in gene expression during development that impact the variability of cell death in retinas treated with NMDA. Interestingly, injection of IL1 $\beta$ prior to NMDA treatment failed to provide neuroprotection in IL-1R1-null retinas. Numbers of TUNEL-positive cells were not significantly different in NMDA-damaged IL-1R1-null retinas treated with vehicle versus treatment with IL1 $\beta$ prior to damage (Fig. $8 \mathrm{a}-\mathrm{d}$ ). However, in retinas from GFAP-Cre-IL-1R1r/r mice, levels of cell death in NMDA-damaged retinas were not significantly different from those observed in WT retinas (Fig. 8ad). In addition, the restoration of IL-1R1expression in astrocytes was sufficient to convey IL1 $\beta$-mediated neuroprotection against NMDA-induced damage. Numbers of TUNEL-positive cells were significantly reduced by treatment with IL1 $\beta$ before NMDA-damage in retinas where IL-1R1 was restored only in astrocytes (Fig. 8a-c). Numbers of dying cells observed inGFAPCre-IL-1R1r/r retinas treated with IL1 $\beta$ were not significantly different from those observed in WT retinas treated with IL1 $\beta$ (Fig. $8 \mathrm{a}-\mathrm{d})$. Further studies are required to determine whether IL1 $\beta /$ IL-1R1-mediated neuroprotection involves IL-1R1 in bipolar cells, endothelial cells, and/or Müller glia.

\section{Discussion}

We report here that microglia can provide protection to retinal neurons following an acute excitotoxic injury. After NMDA-induced damage, microglia acquire an amoeboid morphology and upregulate F4/80, indicative of an "activated" phenotype $[3,49]$. Depletion of microglia via clodronate liposomes or Csf1r-antagonist prior to an excitotoxic insult resulted in increased cell death and decreased neuronal survival. By comparison, treatment of retinas with
IL1 $\beta$ stimulated the reactivity and proliferation of microglia. This "priming" of inflammation with IL1 $\beta$ resulted in decreased cell death and increased neuronal survival following an excitotoxic insult, and the protective effects of IL1 $\beta$ do not require the presence of microglia. These pro-inflammatory cytokines are exclusively expressed by microglia in damaged retinas. Inner retinal neurons, endothelial cells, astrocytes, and Müller glia express components of the IL1-signal transduction pathway and the IL-1R1 receptor.IL1 $\beta$ fails to convey neuroprotection in the IL-1R1-null retina, but neuroprotection is conveyed by IL1 $\beta$ if the expression of IL-1R1 is selectively restored in astrocytes. Taken together, our data support the hypothesis that reactive microglia can provide protection to neurons and this may be mediated, in part, by IL1ßacting at IL-1R1 expressed by astrocytes within the retina.

Microglia become "activated" during the progression of neurodegenerative diseases, but this may not always convey negative consequences. Selective ablation of microglia in an Alzheimer's mouse model resulted in no differences in plaque load or neural survival [50]. Ablation of microglia had no effect on the survival or outgrowth of ganglion cells axons following optic nerve crush, although the depletion of microglia affected the removal of cellular debris [51]. In other contexts, activation of microglia has been associated with the onset of pathology, and suppression of microglial activation is a common strategy to promote neuronal survival and slow disease progression $[4,12,15]$. However, microglia activation is not exclusively correlated with detrimental outcomes $[18,52]$. Microglia have the capacity to release anti-inflammatory cytokines and neurotrophic growth factors [53]. Furthermore, microglia can coordinate activities with Müller glia to mediate neuroprotection in the retina $[48,54]$. Müller glia play a critical role in the context of retinal injury and can be beneficial or harmful to neuronal survival depending on the context [55]. Müller glia can communicate with microglia via diazepam-binding inhibitor ligand (DBI) that activates the translocator protein (TSPO) receptor on microglia [33]. DBI-TSPO signaling is upregulated during injury and inflammation and provides a mechanism for Müller glia to regulate microglia-mediated inflammation [33]. In response to activated microglia, Müller glia can release growth factors such as ciliary neurotrophic factor (CNTF) and leukemia inhibitory factor (LIF), both of 


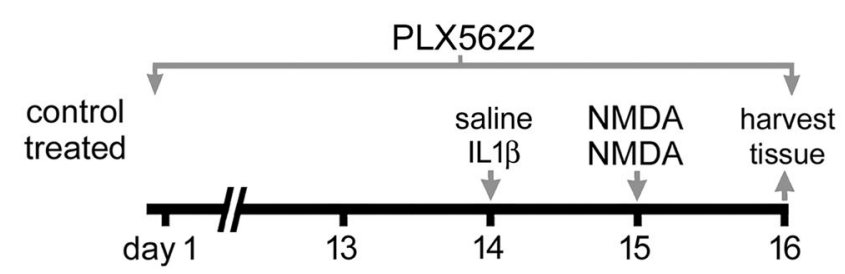

\section{a}
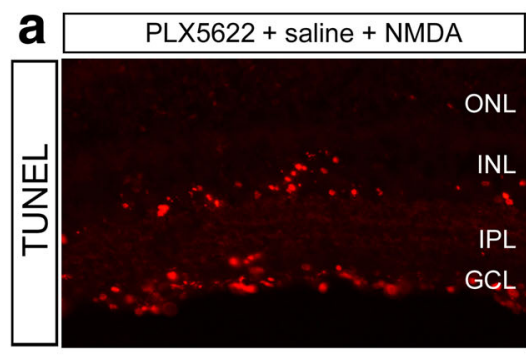

PLX5622 + IL1 $\beta$ + NMDA
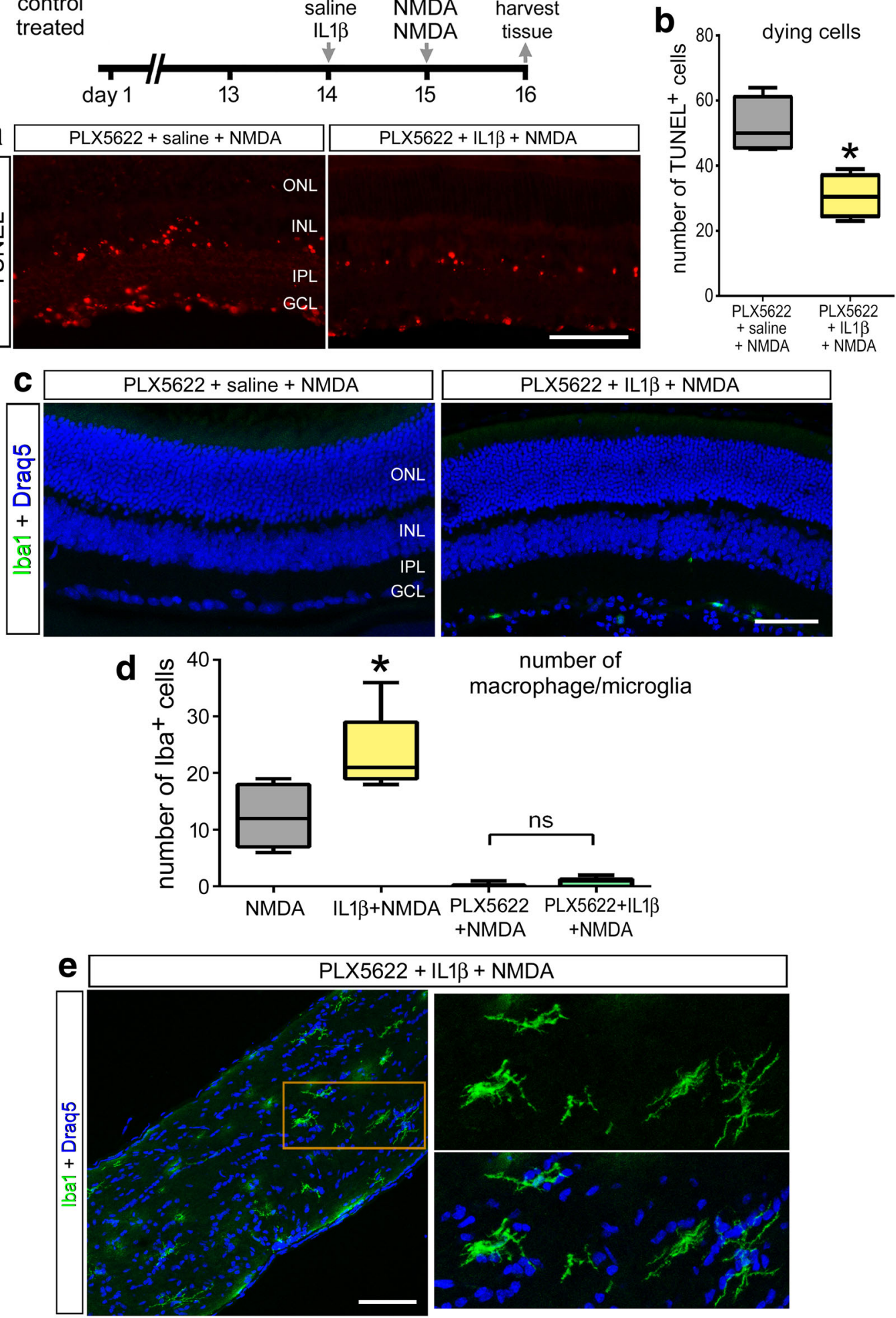

Fig. 7 (See legend on next page.) 
(See figure on previous page.)

Fig. 7 IL1 $\beta$ decreases cell death following NMDA-induced damage in PLX5622-treated retinas. Retinas were obtained from mice that received a diet containing PLX5622 for 2 weeks prior to a single intraocular injection of saline or IL1 $\beta$ at day 14 followed by a single injection of NMDA at day 15, and tissues harvested at day 16 (a-e). Sections of the retina were labeled using TUNEL (a) or antibodies to Iba1 (green; $\mathbf{c}$, e). The boxplots in $\mathbf{b}$ and $\mathbf{d}$ illustrate the mean, upper extreme, lower extreme, upper quartile, and lower quartile ( $n \geq 6$ animals). Images were obtained from central regions of the retina $(\mathbf{a}, \mathbf{c})$ and within the optic nerve $(\mathbf{e})$.Significance of difference $\left(^{*} p<0.05\right)$ between treatment groups was determined using a $t$ test. The calibration bars panels $\mathbf{a}, \mathbf{c}$, and $\mathbf{e}$ represent $50 \mu \mathrm{m}$. Abbreviations: $\mathrm{ONL}$, outer nuclear layer; INL, inner nuclear layer; $\mathrm{IPL}$, inner plexiform layer; $\mathrm{GCL}$, ganglion cell layer

which have been implicated in neuroprotection [56]. The data presented here indicate that the depletion of microglia results in increased death of inner retinal neurons following NMDA-induced excitotoxicity.

Consistent with the notion that reactive microglia can provide protection against excitotoxic damage, we found that treating retinas with IL1 $\beta$ prior to injury leads to a decrease in cell death and an increase in long-term neuronal survival. IL1 $\beta$-stimulated microglia proliferation and F4/ 80 upregulation, indicating that microglia were "activated" by this pro-inflammatory cytokine. IL-1 has been suggested to be a "master regulator" of neuroinflammation and is known to be produced by activated microglia as well as stimulate microglial activation $[57,58]$. Inhibition of IL1 $\beta$ can decrease microglial reactivity, which correlates to a reduction in photoreceptor apoptosis and preservation of retinal function in a mouse model of retinitis pigmentosa [12]. Intraocular injections of tamoxifen are potently neuroprotective and may act by reducing the production of pro-inflammatory cytokines, including IL1 $\beta$ [59]. In contrast to these findings, we find that IL1 $\beta$ application is beneficial to neuronal survival after excitotoxic damage. This discrepancy could be due to the fact that NMDA-injury is an acute damage paradigm and an initial inflammatory response may be helpful to resolve the damaged environment whereas models of photoreceptor disease may represent a model of sustained injury where prolonged inflammation is detrimental. In addition to stimulating microglial reactivity, our data indicate that IL1 $\beta$ may act at different types of retinal cells that express IL-1R1. Interestingly, we find that IL1 $\beta$ treatment is sufficient to activate microglia (Fig. 6), although microglia do not express IL1R1 (Figs. 4 and 5) This is consistent with previous studies indicating the IL1R1 is not expressed by microglia $[21,60]$. These studies indicate that IL1 $\beta$ indirectly activates microglia by acting through IL-1R1. Liu et al. [21] showed that endothelial IL-1R1 mediates IL-1-induced activation of microglia and upregulation of inflammatory mediators. Similarly, Krasnow et al. [60] showed that IL1 $\beta$-treatment results in microglial activation when co-cultured with endothelial cells or astrocytes, whereas IL1 $\beta$ fails to stimulate microglia when cultured alone. Similarly, we find that the survival-influencing effects of IL1 $\beta$ are mediated by signaling through IL-1R1 expressed by astrocytes. Astrocytes have been shown to take on reactive phenotypes that can be neurotoxic or neuroprotective. IL1 $\alpha, \mathrm{TNF} \alpha$, and C1q, secreted from microglia, have been shown to induce an $\mathrm{A} 1$, neurotoxic astrocyte phenotype that promotes neuronal death [61]. IL1 $\beta$ alone or combined with TNF $\alpha$ or C1q favors an A2, neuroprotective astrocyte phenotype [61]. Additionally, recent work has shown that IL1 $\beta$ activation of IL1R1 on astrocytes specifically represses IL-1-induced inflammatory cytokine expression in microglia [21]. Consistent with this notion, significant attention has recently focused upon retinal astrocytes as promising targets to provide neuroprotection to ganglion cells in models of glaucoma [48, 62]. Herein, we may have provided the first direct evidence that a cytokine directly acting at retinal astrocytes impacts the survival of ganglion cells in damaged retina.

It remains unknown whether the signaling from microglia to Müller glia influences neuronal survival in the retina. A previous report found that pharmacological inhibition of $\mathrm{NF} k \mathrm{~B}$ was neuroprotective in NMDA-damaged mouse retinas, and this resulted from decreased production of TNFa by Müller glia [63]. Production of TNF $\alpha$ by Müller glia was probed by immunofluorescence [63]. However, these findings are not consistent with our scRNA-seq data which indicate that $T n f \alpha$ is highly expressed by microglia, but is not expressed by Müller glia in damaged mouse retina (see Fig. 4). Similarly, RNA-seq data from sorted cells from human and mouse brain indicate that Tnfo is highly expressed by microglia/macrophage, but is not expressed by astrocytes, neurons, oligodendrocytes, or endothelial cells $[10,64]$.The influence of NFkB-signaling on neuronal survival may be context-specific and remains controversial with findings supporting both protective [65-67] and detrimental functions $[63,68]$. Further studies are required to determine the sites of action of IL1 $\alpha$ and TNF $\alpha$ in damaged retinas.

NMDA elicits neuronal death via excitotoxicity and over-activation of calcium-permeable ionotropic NMDA receptors [69]. Elevated glutamate can lead to excitotoxicity in retinal pathologies including glaucoma, diabetic retinopathy, and retinal ischemia (reviewed by [70]). Considering our findings that microglia provide neuroprotection following an excitotoxic insult, targeting microglia may be a useful therapeutic strategy in diseases where excitotoxicity plays a role. Most therapeutic strategies targeting microglia in the CNS are aimed at dampening the pro-inflammatory "reactive" microglial responses that occur during chronic 


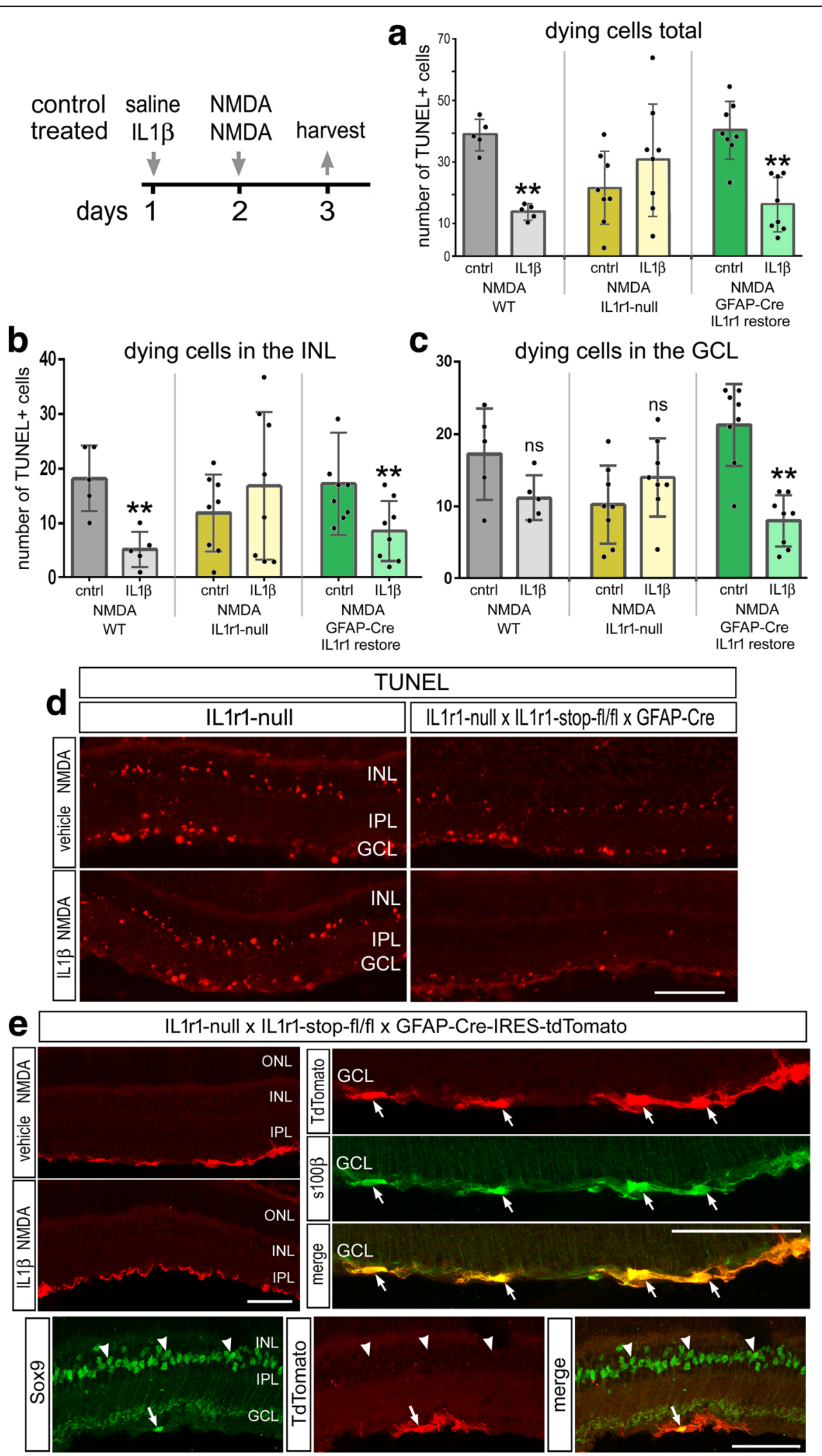

Fig. 8 (See legend on next page.) 
(See figure on previous page.)

Fig. 8 Cell death in IL-1R1-null and GFAPCre-IL-1R1r/r. Eyes were injected with saline or IL1 $\beta$ on day 1, NMDA on day 2, and retinas harvested 1 day later. Experiments were performed on wild-type (WT), IL-1R1-null, andGFAPCre-IL-1R1r/r. The histograms illustrate the mean ( \pm SD and individual data points) number of dying cells across all layers of the retina (a), in the INL (b), and in the GCL (c). Retinal sections were labeled using the TUNEL method (d) or antibodies to RFP (red) and S100ß or Sox9 (green) (e). Significance of difference $(p<0.0001)$ among the treatment groups was determined by one-way ANOVA. Significance of difference $\left({ }^{*} p<0.05\right)$ between treatment groups was determined using a Tukey's multiple comparison test. The calibration bars in panels $\mathbf{d}$ and e represent $50 \mu \mathrm{m}$. Abbreviations: ONL, outer nuclear layer; INL, inner nuclear layer; IPL, inner plexiform layer; GCL, ganglion cell layer

and progressive diseases [4]. Further studies are required to determine how reactive microglia influence neuronal survival following acute or progressive retinal damage in different models of disease. Consistent with our findings, a recent paper by Okunuki et al. [16] demonstrated that depletion of retinal microglia resulted in increased death of photoreceptors following retinal detachment. However, our findings differ significantly with those of Takeda and colleagues wherein ablation of microglia resulted in improved neuronal survival in NMDA-damaged retinas [40]. The differences between our studies may have resulted from differences in doses of NMDA, levels of damage, and activation/ recruitment of microglia/monocytes and their focus on $\mathrm{Brn}^{+}$retinal ganglion cells rather than surveying survival across different types of retinal neurons.

We did not observe significant repopulation of retinal microglia following depletion, likely because we did not make observations following withdrawal of PLX5622. Sources of repopulating microglia are known to include monocytes from the optic nerve [71]. Consistent with this notion, we found Iba1-positive monocytes in the optic nerve following PLX5622-treatment which was maintained through the course of our experiments. We failed to find significant numbers of repopulating monocytes within the retina through the course of our studies. Thus, it is unlikely that reactive, residual, or infiltrating monocytes impacted the diminished survival we observed in PLX5622/NMDA-treated retinas, nor influenced the survival-promoting effects of IL1 $\beta$ in PLX5622/NMDA-treated retinas. Evidence suggests pathogenic roles of microglia and infiltrating macrophages in mouse models of retinal degeneration [72, 73]. By comparison, a recent study has suggested that the protective actions of microglia include recruitment of peripheral monocytes to support the survival of photoreceptors following a detachment injury [16]. The different roles of these two populations of immune cells remain unclear, as microglia and infiltrating macrophages share many immunological functions and recent studies indicate similar genetic profiles between native and repopulated microglia/monocytes [71].

\section{Conclusions}

We conclude that reactive microglia can support survival of retinal neurons following excitotoxic injury, whereas the absence of microglia can be detrimental. In the retina, microglia are the predominant source of IL1 $\beta$, which can act at IL-1R1 which is expressed by astrocytes, endothelial cells, Müller glia, and some inner retinal neurons. We find that exogenous IL1 $\beta$ is neuroprotective against excitotoxic damage, and this effect is predominantly mediated through IL-1R1 expression by retinal astrocytes. Thus, the influence of cytokines upon retinal astrocytes is implicated as an important therapeutic target to impact the survival of retinal neurons, particularly ganglion cells, in diseased retinas.

\section{Additional files}

\begin{abstract}
Additional file 1: Figure S1. Expression of Tspo and Dbi in retinal cells following NMDA-treatment. scRNA-seq was used to identify patterns of expression of Tspo and Dbi in dissociated retinal cells. Each dot represents one cell. t-SNE plots for the expression of Tspo (a) and Dbi (b). Violin/scatter plots of expression of Tspo and Dbi in microglia (c) and Müller glia (d) at different times after NMDA-treatment. The expression of Tspo is prevalent and upregulated in microglia and Müller glia damaged retinas. The expression of Dbi is prevalent in Müller glia and in microglia in damaged retinas. (JPG $3620 \mathrm{~kb}$ )
\end{abstract}

Additional file 2: Figure S2. Expression of Tnfrsf isoforms in retinal cells following NMDA-treatment. scRNA-seq was used to identify patterns of expression of Tnfrsf isoforms in dissociated retinal cells. Each dot represents one cell. Violin/scatter plots of expression of Tnfrsf isoforms in microglia and Müller glia at different times after NMDA-treatment. (JPG $1160 \mathrm{~kb})$

Additional file 3: Figure S3. Expression of TIr isoforms in retinal cells following NMDA-treatment. scRNA-seq was used to identify patterns of expression of T/r isoforms among acutely dissociated retinal cells. Each dot represents one cell. Cells were sampled from control retinas (rep1 5300 cells and rep2 12,932 cells) and from retinas at $3 \mathrm{~h}$ (8518 cells), $6 \mathrm{~h}$ (8000 cells), 12 h (4307 cells), 24 h (4270 cells), 36 h (1618 cells), 48 h (2246 cells), and $72 \mathrm{~h}$ ( 2269 cells) after NMDA-treatment (a). tSNE plots revealed distinct clustering of different types of retinal cells and numbers of cells surveyed (in parentheses) (b). Microglia were identified based on collective expression of Rgs1, Trem2, CCl3, CCl4, Clqa, Clab, and Clqc (Fig. 4d). (c) t-SNE plots for the collective expression of TIr1, TIr4, T/r7 and TIr13; expression is predominantly restricted to microglia. (d, e) t-SNE plots for the expression of T/r2 and T/r3. Violin/scatter plots of expression of T/r isoforms in Müller glia at different times after NMDA-treatment ( $f$ ). (JPG $3820 \mathrm{~kb}$ )

Additional file 4: Figure S4. IL-1R1-HA is localized to astrocytes near the vitread surface of the retinas. Sections of the retina were labeled for HA-immunoreactivity in both IL-1R1-3HA-IRES-tdTomato mice and GFAPCre-IL-1R1r/r mice, which also contain the IL-1R1-3HA-IRES-tdTomato sequence. Abbreviations: $\mathrm{ONL}$, outer nuclear layer; INL, inner nuclear layer; IPL, inner plexiform layer; GCL, ganglion cell layer. (JPG 3120 kb)

\section{Abbreviations}

ANOVA: Analysis of variance; CNS: Central nervous system; CNTF: Ciliary neurotrophic factor; CSF1R: Colony-stimulating factor 1 receptor; DBI: Diazepam-binding inhibitor ligand; GCL: Ganglion cell layer; GFAP: Glial fibrillary acidic protein; IL1R1: Interleukin 1 receptor type 1; IL 1 3 : Interleukin 1 
beta; INL: Inner nuclear layer; IRAKs: Interleukin-1 receptor-associated kinases; IRES: Internal ribosomal entry site; LIF: Leukemia inhibitory factor; NFkB: Nuclear factor kappa B; NMDA: N-methyl-D-aspartate; RFP: Red fluorescent protein; RPE: Retinal pigmented epithelium; scRNA-seq: Singlecell RNA sequencing; TNFa: Tumor necrosis factor alpha; tSNE: $t$-Distributed Stochastic Neighbor Embedding; TSPO: Translocator protein; TUNEL: Terminal deoxynucleotidyl transferase dUTP nick end labeling; WT: Wild type

\section{Acknowledgements}

Not applicable.

\section{Funding}

This work was supported by RO1 EY022030-06 (AJF), UO1 EY027267-02(SB, AJF), and R01 MH109165-01 (NQ).

\section{Availability of data and materials}

The datasets used and/or analyzed during the current study are available from the corresponding author on reasonable request.

\section{Authors' contributions}

LT and IP designed and executed experiments, gathered data, constructed figures, and contributed to writing the manuscript. LS, LV, WAC, and TH executed experiments, gathered data, and constructed figures. AJF, NQ, and SB designed experiments, constructed figures, and contributed to writing the manuscript. All authors read and approved the final manuscript.

\section{Ethics approval and consent to participate}

The use of animals in these experiments was in accordance with the guidelines established by the National Institutes of Health and the Institutional Animal Care and Use Committee at Ohio State University.

\section{Consent for publication}

Not applicable.

\section{Competing interests}

The authors declare that they have no competing interests.

\section{Publisher's Note}

Springer Nature remains neutral with regard to jurisdictional claims in published maps and institutional affiliations.

\section{Author details}

${ }^{1}$ Department of Neuroscience, College of Medicine, The Ohio State University, 3020 Graves Hall, 333 W. 10th Ave, Columbus, OH 43210-1239, USA. ${ }^{2}$ Department of Pathology and Immunology, Washington University School of Medicine, St. Louis, MO, USA. 3Solomon H. Snyder Department of Neuroscience, Johns Hopkins University School of Medicine, Baltimore, MD, USA. ${ }^{4}$ Institute for Behavioral Medicine Research, College of Medicine, The Ohio State University, Columbus, OH, USA. ${ }^{5}$ Division of Biosciences, College of Dentistry, The Ohio State University, Columbus, OH, USA. ${ }^{6}$ Department of Biological Structure, University of Washington, Seattle, WA, USA

\section{Received: 31 January 2019 Accepted: 9 May 2019}

\section{Published online: 06 June 2019}

\section{References}

1. Aguzzi A, Barres BA, Bennett ML. Microglia: scapegoat, saboteur, or something else? Science. 2013;339(6116):156-61.

2. Ginhoux F, Greter M, Leboeuf M, Nandi S, See P, Gokhan S, et al. Fate mapping analysis reveals that adult microglia derive from primitive macrophages. Science. 2010;330(6005):841-5.

3. Santos AM, Calvente R, Tassi M, Carrasco MC, Martin-Oliva D, Marin-Teva JL, et al. Embryonic and postnatal development of microglial cells in the mouse retina. J Comp Neurol. 2008:506(2):224-39.

4. Karlstetter M, Scholz R, Rutar M, Wong WT, Provis JM, Langmann T. Retinal microglia: just bystander or target for therapy? Prog Retin Eye Res. 2015;45:30-57.

5. Schafer DP, Lehrman EK, Kautzman AG, Koyama R, Mardinly AR, Yamasaki R, et al. Microglia sculpt postnatal neural circuits in an activity and complement-dependent manner. Neuron. 2012;74(4):691-705.
6. Nimmerjahn A, Kirchhoff F, Helmchen F. Resting microglial cells are highly dynamic surveillants of brain parenchyma in vivo. Science. 2005; 308(5726):1314-8

7. Chawla A, Nguyen KD, Goh YP. Macrophage-mediated inflammation in metabolic disease. Nat Rev Immunol. 2011;11(11):738-49.

8. Li Q, Barres BA. Microglia and macrophages in brain homeostasis and disease. Nat Rev Immunol. 2018;18(4):225-42.

9. Ransohoff RM, Cardona AE. The myeloid cells of the central nervous system parenchyma. Nature. 2010;468(7321):253-62.

10. Zhang Y, Chen K, Sloan SA, Bennett ML, Scholze AR, O'Keeffe S, et al. An RNA-sequencing transcriptome and splicing database of glia, neurons, and vascular cells of the cerebral cortex. J Neurosci. 2014;34(36):11929-47.

11. Block ML, Zecca L, Hong JS. Microglia-mediated neurotoxicity: uncovering the molecular mechanisms. Nat Rev Neurosci. 2007;8(1):57-69.

12. Zhao L, Zabel MK, Wang X, Ma W, Shah P, Fariss RN, et al. Microglial phagocytosis of living photoreceptors contributes to inherited retinal degeneration. EMBO Mol Med. 2015;7(9):1179-97.

13. Stevens B, Allen NJ, Vazquez LE, Howell GR, Christopherson KS, Nouri N, et al. The classical complement cascade mediates CNS synapse elimination. Cell. 2007;131(6):1164-78.

14. Williams PA, Tribble JR, Pepper KW, Cross SD, Morgan BP, Morgan JE, et al. Inhibition of the classical pathway of the complement cascade prevents early dendritic and synaptic degeneration in glaucoma. Mol Neurodegener. 2016;11:26

15. Zhao L, Ma W, Fariss RN, Wong WT. Minocycline attenuates photoreceptor degeneration in a mouse model of subretinal hemorrhage microglial: inhibition as a potential therapeutic strategy. Am J Pathol. 2011;179(3):1265-77.

16. Okunuki $Y$, Mukai R, Pearsall EA, Klokman G, Husain D, Park DH, et al. Microglia inhibit photoreceptor cell death and regulate immune cell infiltration in response to retinal detachment. Proc Natl Acad Sci U S A. 2018;115(27):E6264-73

17. Fischer AJ, Zelinka C, Milani-Nejad N. Reactive retinal microglia, neurona survival, and the formation of retinal folds and detachments. Glia. 2015; 63(2):313-27.

18. Vinet J, Weering HR, Heinrich A, Kalin RE, Wegner A, Brouwer $N$, et al. Neuroprotective function for ramified microglia in hippocampal excitotoxicity. J Neuroinflammation. 2012;9:27.

19. Rice RA, Spangenberg EE, Yamate-Morgan H, Lee RJ, Arora RP, Hernandez MX, et al. Elimination of microglia improves functional outcomes following extensive neuronal loss in the hippocampus. J Neurosci. 2015;35(27):9977-89.

20. Song A, Zhu L, Gorantla G, Berdysz O, Amici SA, Guerau-de-Arellano M, et al. Salient type 1 interleukin 1 receptor expression in peripheral non-immune cells. Sci Rep. 2018;8(1):723

21. Liu X, Nemeth DP, McKim DB, Zhu L, DiSabato DJ, Berdysz O, et al. Celltype-specific interleukin 1 receptor 1 signaling in the brain regulates distinct Neuroimmune activities. Immunity. 2019;50(2):317-33.

22. Zelinka CP, Scott MA, Volkov L, Fischer AJ. The reactivity, distribution and abundance of non-astrocytic inner retinal glial (NIRG) cells are regulated by microglia, acute damage, and IGF1. PLoS One. 2012;7(9): e44477

23. Van Rooijen N. The liposome-mediated macrophage 'suicide' technique. J Immunol Methods. 1989;124(1):1-6.

24. Powers AN, Satija R. Single-cell analysis reveals key roles for Bcl11a in regulating stem cell fate decisions. Genome Biol. 2015;16:199.

25. Satija R, Farrell JA, Gennert D, Schier AF, Regev A. Spatial reconstruction of single-cell gene expression data. Nat Biotechnol. 2015;33(5):495-502.

26. Fischer AJ, Foster S, Scott MA, Sherwood P. Transient expression of LIMdomain transcription factors is coincident with delayed maturation of photoreceptors in the chicken retina. J Comp Neurol. 2008;506(4):584-603.

27. Fischer AJ, Zelinka C, Gallina D, Scott MA, Todd L. Reactive microglia and macrophage facilitate the formation of Muller glia-derived retinal progenitors. Glia. 2014;62(10):1608-28

28. Gallina D, Zelinka CP, Cebulla CM, Fischer AJ. Activation of glucocorticoid receptors in Muller glia is protective to retinal neurons and suppresses microglial reactivity. Exp Neurol. 2015;273:114-25.

29. Kuehn S, Rodust C, Stute G, Grotegut P, Meissner W, Reinehr S, et al. Concentration-dependent inner retina layer damage and optic nerve degeneration in a NMDA model. J Mol Neurosci. 2017;63(3-4):283-99.

30. Santos AM, Martin-Oliva D, Ferrer-Martin RM, Tassi M, Calvente R, Sierra A, et al. Microglial response to light-induced photoreceptor degeneration in the mouse retina. J Comp Neurol. 2010;518(4):477-92. 
31. Almolda B, de Labra C, Barrera I, Gruart A, Delgado-Garcia JM, Villacampa N, et al. Alterations in microglial phenotype and hippocampal neuronal function in transgenic mice with astrocyte-targeted production of interleukin-10. Brain Behav Immun. 2015:45:80-97.

32. Karlstetter M, Nothdurfter C, Aslanidis A, Moeller K, Horn F, Scholz R, et al. Translocator protein (18 kDa) (TSPO) is expressed in reactive retinal microglia and modulates microglial inflammation and phagocytosis. J Neuroinflammation. 2014;11:3.

33. Wang M, Wang X, Zhao L, Ma W, Rodriguez IR, Fariss RN, et al. Macrogliamicroglia interactions via TSPO signaling regulates microglial activation in the mouse retina. J Neurosci. 2014;34(10):3793-806.

34. Mages K, Grassmann F, Jagle H, Rupprecht R, Weber BHF, Hauck SM, et al. The agonistic TSPO ligand XBD173 attenuates the glial response thereby protecting inner retinal neurons in a murine model of retinal ischemia. J Neuroinflammation. 2019;16(1):43.

35. Elmore MR, Najafi AR, Koike MA, Dagher NN, Spangenberg EE, Rice RA, et al. Colony-stimulating factor 1 receptor signaling is necessary for microglia viability, unmasking a microglia progenitor cell in the adult brain. Neuron. 2014:82(2):380-97.

36. Dharmarajan S, Fisk DL, Sorenson CM, Sheibani N, Belecky-Adams TL. Microglia activation is essential for BMP7-mediated retinal reactive gliosis. J Neuroinflammation. 2017;14(1):76.

37. Dinarello CA, Simon A, van der Meer JW. Treating inflammation by blocking interleukin-1 in a broad spectrum of diseases. Nat Rev Drug Discov. 2012; 11(8):633-52.

38. Tartaglia LA, Goeddel DV. Two TNF receptors. Immunol Today. 1992;13(5):151-3.

39. Brockhaus M, Schoenfeld HJ, Schlaeger EJ, Hunziker W, Lesslauer W, Loetscher $\mathrm{H}$. Identification of two types of tumor necrosis factor receptors on human cell lines by monoclonal antibodies. Proc Natl Acad Sci U S A. 1990;87(8):3127-31.

40. Takeda A, Shinozaki Y, Kashiwagi K, Ohno N, Eto K, Wake H, et al. Microglia mediate non-cell-autonomous cell death of retinal ganglion cells. Glia. 2018; 66(11):2366-84.

41. Bennett ML, Bennett FC, Liddelow SA, Ajami B, Zamanian JL, Fernhoff NB, et al. New tools for studying microglia in the mouse and human CNS. Proc Natl Acad Sci U S A. 2016:113(12):E1738-46.

42. Peng YR, Shekhar K, Yan W, Herrmann D, Sappington A, Bryman GS, et al. Molecular classification and comparative Taxonomics of foveal and peripheral cells in primate retina. Cell. 2019;176(5):1222-37 e22.

43. Lin SC, Lo YC, Wu H. Helical assembly in the MyD88-IRAK4-IRAK2 complex in TLR/IL-1R signalling. Nature. 2010;465(7300):885-90

44. Xu Y, Tao X, Shen B, Horng T, Medzhitov R, Manley JL, et al. Structural basis for signal transduction by the toll/interleukin-1 receptor domains. Nature. 2000;408(6808):111-5.

45. Burns $K$, Martinon F, Esslinger $C$, Pahl H, Schneider P, Bodmer JL, et al. MyD88, an adapter protein involved in interleukin-1 signaling. J Biol Chem. 1998:273(20):12203-9.

46. Cuenca N, Fernandez-Sanchez L, Campello L, Maneu V, De la Villa P, Lax P, et al. Cellular responses following retinal injuries and therapeutic approaches for neurodegenerative diseases. Prog Retin Eye Res. 2014;43:17-75.

47. Karlstetter M, Ebert S, Langmann T. Microglia in the healthy and degenerating retina: insights from novel mouse models. Immunobiology. 2010;215(9-10):685-91.

48. Vecino E, Rodriguez FD, Ruzafa N, Pereiro X, Sharma SC. Glia-neuron interactions in the mammalian retina. Prog Retin Eye Res. 2016;51:1-40.

49. Bye N, Habgood MD, Callaway JK, Malakooti N, Potter A, Kossmann T, et al. Transient neuroprotection by minocycline following traumatic brain injury is associated with attenuated microglial activation but no changes in cell apoptosis or neutrophil infiltration. Exp Neurol. 2007;204(1):220-33.

50. Grathwohl SA, Kalin RE, Bolmont T, Prokop S, Winkelmann G, Kaeser SA, et al. Formation and maintenance of Alzheimer's disease beta-amyloid plaques in the absence of microglia. Nat Neurosci. 2009;12(11):1361-3.

51. Hilla AM, Diekmann H, Fischer D. Microglia are irrelevant for neuronal degeneration and axon regeneration after acute injury. J Neurosci. 2017; 37(25):6113-24.

52. Chen Z, Jalabi W, Shpargel KB, Farabaugh KT, Dutta R, Yin X, et al. Lipopolysaccharide-induced microglial activation and neuroprotection against experimental brain injury is independent of hematogenous TLR4. J Neurosci. 2012;32(34):11706-15.

53. Saijo K, Glass CK. Microglial cell origin and phenotypes in health and disease. Nat Rev Immunol. 2011;11(11):775-87.
54. Wang M, Wong WT. Microglia-Muller cell interactions in the retina. Adv Exp Med Biol. 2014:801:333-8.

55. Bringmann A, landiev I, Pannicke T, Wurm A, Hollborn M, Wiedemann P, et al. Cellular signaling and factors involved in Muller cell gliosis: neuroprotective and detrimental effects. Prog Retin Eye Res. 2009;28(6):423-51.

56. Wang M, Ma W, Zhao L, Fariss RN, Wong WT. Adaptive Muller cell responses to microglial activation mediate neuroprotection and coordinate inflammation in the retina. J Neuroinflammation. 2011;8:173.

57. Basu A, Krady JK, Levison SW. Interleukin-1: a master regulator of neuroinflammation. J Neurosci Res. 2004;78(2):151-6.

58. Basu A, Krady JK, Enterline JR, Levison SW. Transforming growth factor beta1 prevents IL-1 beta-induced microglial activation, whereas TNFalphaand IL-6-stimulated activation are not antagonized. Glia. 2002;40(1):109-20.

59. Wang X, Zhao L, Zhang Y, Ma W, Gonzalez SR, Fan J, et al. Tamoxifen provides structural and functional rescue in murine models of photoreceptor degeneration. J Neurosci. 2017;37(12):3294-310.

60. Krasnow SM, Knoll JG, Verghese SC, Levasseur PR, Marks DL. Amplification and propagation of interleukin-1 beta signaling by murine brain endothelial and glial cells. J Neuroinflammation. 2017;14(1):133.

61. Liddelow SA, Guttenplan KA, Clarke LE, Bennett FC, Bohlen CJ, Schirmer L, et al. Neurotoxic reactive astrocytes are induced by activated microglia. Nature. 2017:541(7638):481-7.

62. Levin LA, Crowe ME, Quigley HA, Lasker IIA, Glaucomatous Neurodegeneration P. Neuroprotection for glaucoma: requirements for clinical translation. Exp Eye Res. 2017;157:34-7.

63. Lebrun-Julien F, Duplan L, Pernet V, Osswald I, Sapieha P, Bourgeois P, et al. Excitotoxic death of retinal neurons in vivo occurs via a non-cellautonomous mechanism. J Neurosci. 2009;29(17):5536-45.

64. Zhang Y, Sloan SA, Clarke LE, Caneda C, Plaza CA, Blumenthal PD, et al. Purification and characterization of progenitor and mature human astrocytes reveals transcriptional and functional differences with mouse Neuron. 2016:89(1):37-53.

65. Mattson MP. NF-kappaB in the survival and plasticity of neurons. Neurochem Res. 2005;30(6-7):883-93.

66. Bhakar AL, Tannis LL, Zeindler C, Russo MP, Jobin C, Park DS, et al. Constitutive nuclear factor-kappa B activity is required for central neuron survival. J Neurosci. 2002;22(19):8466-75.

67. Yu Z, Zhou D, Bruce-Keller AJ, Kindy MS, Mattson MP. Lack of the p50 subunit of nuclear factor-kappaB increases the vulnerability of hippocampal neurons to excitotoxic injury. J Neurosci. 1999;19(20):8856-65.

68. Schneider A, Martin-Villalba A, Weih F, Vogel J, Wirth T, Schwaninger M. NFkappaB is activated and promotes cell death in focal cerebral ischemia. Nat Med. 1999;5(5):554-9.

69. Papadia S, Hardingham GE. The dichotomy of NMDA receptor signaling. Neuroscientist. 2007;13(6):572-9.

70. Ishikawa M. Abnormalities in glutamate metabolism and excitotoxicity in the retinal diseases. Scientifica (Cairo). 2013;2013:528940.

71. Huang $Y, X u$ Z, Xiong S, Qin G, Sun F, Yang J, et al. Dual extra-retinal origins of microglia in the model of retinal microglia repopulation. Cell Discov. 2018:4:9.

72. Peng B, Xiao J, Wang K, So KF, Tipoe GL, Lin B. Suppression of microglial activation is neuroprotective in a mouse model of human retinitis pigmentosa. J Neurosci. 2014;34(24):8139-50.

73. Sennlaub F, Auvynet C, Calippe B, Lavalette S, Poupel L, Hu SJ, et al. CCR2(+ monocytes infiltrate atrophic lesions in age-related macular disease and mediate photoreceptor degeneration in experimental subretinal inflammation in Cx3cr1 deficient mice. EMBO Mol Med. 2013;5(11):1775-93.

Ready to submit your research? Choose BMC and benefit from:

- fast, convenient online submission

- thorough peer review by experienced researchers in your field

- rapid publication on acceptance

- support for research data, including large and complex data types

- gold Open Access which fosters wider collaboration and increased citations

- maximum visibility for your research: over $100 \mathrm{M}$ website views per year

At $\mathrm{BMC}$, research is always in progress.

Learn more biomedcentral.com/submission 